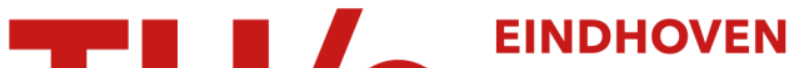

\section{Simultaneous optimization of speed and buffer times with an application to liner shipping}

Citation for published version (APA):

Mulder, J., van Jaarsveld, W., \& Dekker, R. (2019). Simultaneous optimization of speed and buffer times with an application to liner shipping. Transportation Science, 53(2), 365-382. https://doi.org/10.1287/trsc.2018.0842

\section{Document license:}

TAVERNE

DOI:

10.1287/trsc.2018.0842

Document status and date:

Published: 01/03/2019

\section{Document Version:}

Publisher's PDF, also known as Version of Record (includes final page, issue and volume numbers)

\section{Please check the document version of this publication:}

- A submitted manuscript is the version of the article upon submission and before peer-review. There can be important differences between the submitted version and the official published version of record. People interested in the research are advised to contact the author for the final version of the publication, or visit the $\mathrm{DOI}$ to the publisher's website.

- The final author version and the galley proof are versions of the publication after peer review.

- The final published version features the final layout of the paper including the volume, issue and page numbers.

Link to publication

\section{General rights}

Copyright and moral rights for the publications made accessible in the public portal are retained by the authors and/or other copyright owners and it is a condition of accessing publications that users recognise and abide by the legal requirements associated with these rights.

- Users may download and print one copy of any publication from the public portal for the purpose of private study or research.

- You may not further distribute the material or use it for any profit-making activity or commercial gain

- You may freely distribute the URL identifying the publication in the public portal.

If the publication is distributed under the terms of Article $25 \mathrm{fa}$ of the Dutch Copyright Act, indicated by the "Taverne" license above, please follow below link for the End User Agreement:

www.tue.nl/taverne

Take down policy

If you believe that this document breaches copyright please contact us at:

openaccess@tue.nl

providing details and we will investigate your claim. 
This article was downloaded by: [131.155.144.47] On: 10 J uly 2019, At: 04:47

Publisher: Institute for Operations Research and the Management Sciences (INFORMS)

INFORMS is located in Maryland, USA

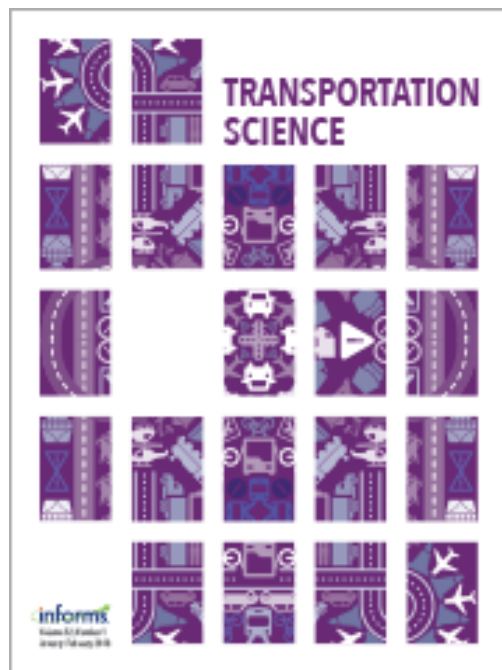

\section{Transportation Science}

Publication details, including instructions for authors and subscription information:

http:// pubsonline.informs. org

\section{Simultaneous Optimization of Speed and Buffer Times with an Application to Liner Shipping}

J udith Mulder, Willem van J aarsveldRommert Dekker

\section{To cite this article:}

J udith Mulder, Willem van J aarsveldRommert Dekker (2019) Simultaneous Optimization of Speed and Buffer Times with an Application to Liner Shipping. Transportation Science 53(2):365-382. https:// doi.org/ 10.1287/ trsc. 2018.0842

\section{Full terms and conditions of use: https://pubsonline.informs.org/page/terms-and-conditions}

This article may be used only for the purposes of research, teaching, and/or private study. Commercial use or systematic downloading (by robots or other automatic processes) is prohibited without explicit Publisher approval, unless otherwise noted. For more information, contact permissions@informs.org.

The Publisher does not warrant or guarantee the article's accuracy, completeness, merchantability, fitness for a particular purpose, or non-infringement. Descriptions of, or references to, products or publications, or inclusion of an advertisement in this article, neither constitutes nor implies a guarantee, endorsement, or support of claims made of that product, publication, or service.

Copyright @ 2019, INFORMS

\section{Please scroll down for article-it is on subsequent pages}

INFORMS is the largest professional society in the world for professionals in the fields of operations research, management science, and analytics.

For more information on INFORMS, its publications, membership, or meetings visit http:// www. informs. org 


\title{
Simultaneous Optimization of Speed and Buffer Times with an Application to Liner Shipping
}

\author{
Judith Mulder, ${ }^{a}$ Willem van Jaarsveld, ${ }^{b}$ and Rommert Dekker ${ }^{a}$ \\ ${ }^{\mathbf{a}}$ Econometric Institute, Erasmus University Rotterdam, 3062 PA Rotterdam, Netherlands; ${ }^{\mathbf{b}}$ School of Industrial Engineering, Eindhoven \\ University of Technology, 5600 MB Eindhoven, Netherlands \\ Contact: jmulder@ret.nl, (D) https://orcid.org/0000-0002-9102-9823 (JM); w.l.v.jaarsveld@tue.nl, \\ (iD https://orcid.org/0000-0003-3620-4067 (WvJ); rdekker@ese.eur.nl, (D) https://orcid.org/0000-0003-3823-1990 (RD)
}

Received: February 9, 2017

Revised: December 20, 2017; March 29, 2018

Accepted: April 4, 2018

Published Online in Articles in Advance: March 26, 2019

https://doi.org/10.1287/trsc.2018.0842

Copyright: ๑) 2019 INFORMS

\begin{abstract}
Transport companies often have a published timetable. To maintain timetable reliability despite delays, companies include buffer times during timetable development and adjust the traveling speed during timetable execution. We develop an approach that integrates timetable development and execution. We model execution of the timetable as a stochastic dynamic program (SDP). An SDP is a natural framework to model random events causing (additional) delay, propagation of delays, and real-time optimal speed adjustments. However, SDPs alone cannot incorporate the buffer allocation during timetable development, as buffer allocation requires choosing the same action in different states of the SDP. Motivated by the practical need for timetables that operate well during timetable execution, our model seeks the buffer allocation that yields the SDP that has minimal long-run average costs. We derive several analytical insights into the model. We prove that costs are joint convex in the buffer times, and we develop theory to compute subgradients. Our fast and exact algorithm for buffer time allocation is based on these results. Our case study considers container vessels sailing a round tour consisting of 14 ports based on Maersk data. The algorithm finds the optimal timetable in roughly 70 seconds for realistic problem instances. The optimal timetable yields cost reductions of about four to 10 million U.S. dollars per route per year in comparison with the current timetable. Finally, we show the robustness of our solution approach for different parameter settings using a sensitivity analysis.
\end{abstract}

Funding: Research by J. Mulder was supported by grants from the the Netherlands Organisation for Scientific Research (NWO) Dinalog Integrated Synchromodal Transport System Analysis (ISOLA) project and the Leiden-Delft-Erasmus Centre for Metropolis and Mainport.

Supplemental Material: The online appendices are available at https://doi.org/10.1287/trsc.2018.0842.

Keywords: robust timetabling • (online) speed optimization • stochastic dynamic programming

\section{Introduction}

Timetables are used in container shipping, airlines, and public transport to communicate planned arrival and departure times in advance to customers. However, delays are inevitable while executing the timetable, making the arrival times uncertain. Maintaining timetable reliability despite these delays is crucial: the timetable is relied on by passengers and freight forwarders. However, unforeseen events may cause delays with respect to the timetable. For example, recent liner shipping statistics show that only $64 \%$ of ships are delayed less than 24 hours with respect to the timetable (CargoSmart 2017).

Transport companies combine two main methods to ensure a reliable schedule. Firstly, during timetable development, a more delay-resistant planning may be obtained by including buffer or slack time. In liner shipping, for example, the planned arrival at the port of Jeddah could be 9.5 days after the planned departure from Rotterdam, while the trip takes only eight days on average when sailing at design speed. The 36 hours of buffer time can capture (part of) a delay. However, buffers increase the nominal travel time and therefore costs. So limited buffer time is available, and optimal allocation along a route is key. Secondly, during execution of the timetable, a ship may sail faster to recover from a delay with respect to the timetable, but increasing speed is very costly: Figure 1 shows that sailing at 24 knots instead of 16 knots approximately doubles fuel consumption per nautical mile (nmi) for an 8,000-20-foot equivalent unit (TEU) ship. For a trip from Rotterdam to Jeddah, this corresponds to a difference of over 200,000 U.S. dollars (USD) at a bunker price of $300 \mathrm{USD} / \mathrm{ton}$, or about 2,125 tons of $\mathrm{CO}_{2}$ (Cariou 2011). Speed adjustments also have significant impact for other transport modes: for example doubling the average speed of a metro on a track roughly quadruples energy consumption (Binder and Albrecht 2012). We will focus on the liner shipping application throughout the paper. The relevance of the developed approach for other transportation modes is discussed in Section 7. 
Figure 1. Fuel (Bunker) Consumption of Several Container Ships at Different Travel Speeds

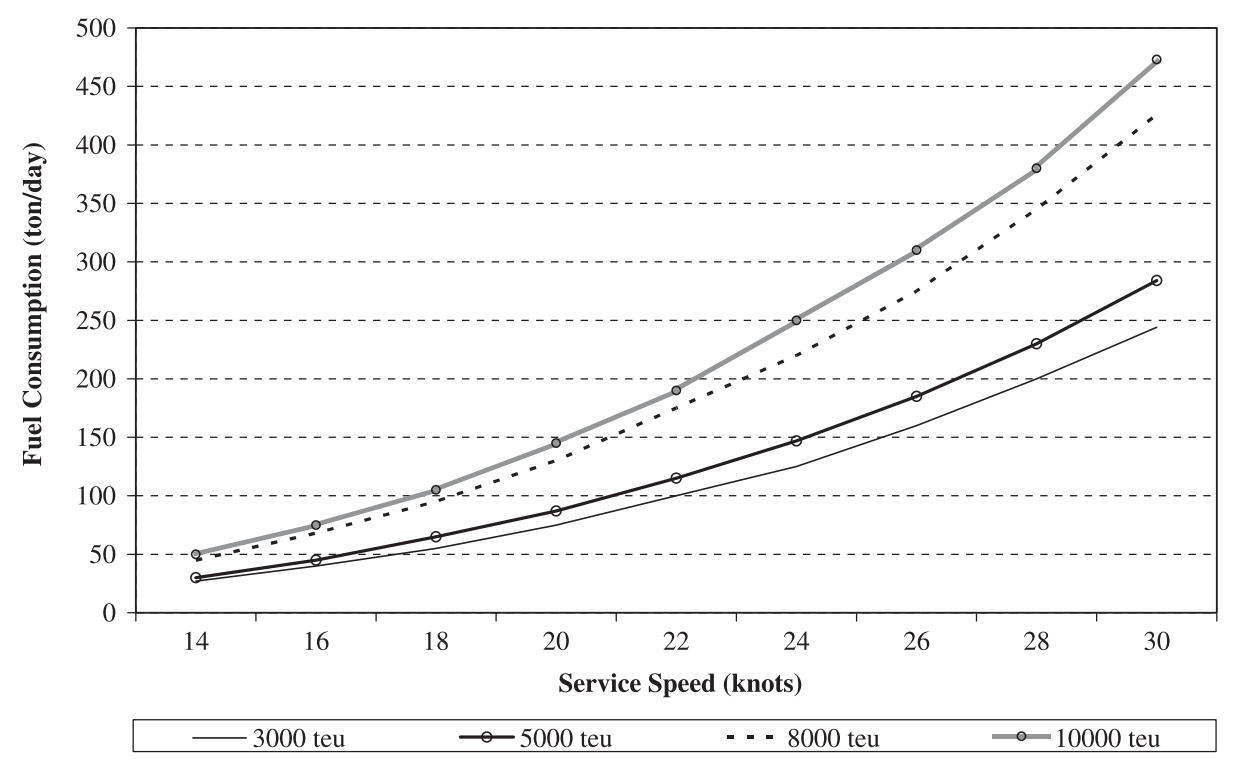

Source. Notteboom and Vernimmen (2009).

Our approach to developing a timetable and determining recovery actions consists of two stages. The first stage involves the allocation of buffers (construction of the timetable), which is a problem with here-and-now decisions on the tactical planning level. We consider the situation in which routes have already been decided on, so the available amount of total buffer time is known and given. In the second stage, the timetable is executed and recourse decisions need to be taken on the operational level: random events cause (additional) delay, travel speed is optimized, and late arrivals and departures are penalized. The optimal recourse actions (speed decisions) may be nonlinear in the delay. Therefore, we model the operational planning level as a stochastic dynamic program (SDP) as this can capture nonlinearity in the speed decisions. Additionally, the SDP accurately models propagation of delays from port call to port call. However, the buffer times are exogenous to this SDP: different buffer time allocations yield SDPs that are structurally different. The optimal buffer allocation yields the SDP that has minimal long-run average costs.

We contribute a theoretical analysis of the problem. For example, we show that speed should increase as the delay with respect to the schedule increases, and provide a bound on the maximum speed increase that should result from additional delay. More importantly, we prove the key result that the minimum costs of operating the timetable are joint convex in the buffer time allocation. Based on this result, we develop further theoretical results to optimally allocate the buffers on the tactical level, taking into account delays and speed optimization (as part of the SDP) on the operational level. These theoretical results lead to a simple and efficient approach for computing subgradients. Our algorithm for optimal buffer time allocation is based on these subgradients. This algorithm finds the optimal solution in less than 75 seconds for realistic problem instances. To the best of our knowledge, the algorithm is the first exact algorithm for timetabling under stochastic uncertainty. We then report on a case study and sensitivity analysis based on Maersk data for a round tour consisting of 14 ports.

The remainder of this paper is organized as follows. In Section 2, we review literature and explain how our work contributes to this literature. Section 3 provides a detailed description of the model. The theoretical analysis of the model is presented in Section 4. In Section 5, we develop the algorithm, and provide further theoretical results underlying the algorithm. Section 6 describes computational experiments. We conclude in Section 7.

\section{Literature Review}

Timetables are often used in air, railway, and maritime transport. Multiple studies have already been performed on managing, recovering, and preventing delays in these transport modes. Clausen et al. (2010) give an overview on disruption management studies in the airline industry. They distinguish the reviewed studies in two different groups: delay recovery and robust planning. The first group optimizes recovery actions (these are online/operational decisions (e.g., speed adjustments)). The second group optimizes timetables/schedules in anticipation of uncertain delays, but before delays occur (offline/tactical decisions). This distinction also mainly holds for railway and maritime transport, and we use it in the literature review below. In deterministic settings, the planned travel time uniquely determines the actual travel speed, and as a consequence the distinction between online and offline 
decisions typically disappears. Literature in deterministic settings is therefore reviewed separately.

\subsection{Deterministic Settings}

Hvattum et al. (2013), Kramer et al. (2015), and Franceschetti (2015) propose polynomial time exact algorithms for deterministic departure time optimization given a fixed route. In these studies, the order in which nodes are visited is fixed, and each node should be visited within a certain time window. Hvattum et al. (2013) propose an exact algorithm to determine the speed on each arc in such a way that all time windows are satisfied and total fuel costs are minimized. Kramer et al. (2015) extend this algorithm to also optimize the departure time from the depot, while Franceschetti (2015) proposes an exact algorithm that optimizes the departure time at each node. Finally, Reinhardt et al. (2016) consider deterministic optimization of speed in a network by adjusting the port berthing times (arrival and departure times). A key advantage of this approach is that it handles transit time requirements, including requirements for container flows that use multiple services. They develop an MIP approach for this problem that ingeniously incorporates the cyclic nature of the services. Their collaboration with Maersk line enables development of test instances representative of real-life networks, and for these instances they find significant potential savings when they allow transit times to increase at most 48 hours compared with the current timetable. Note that the ideas in these deterministic models are complementary to the approach developed in this paper: we focus on dealing with the considerable uncertainties of liner shipping by optimally combining buffer allocation and online recovery actions.

\subsection{Delay Recovery}

Delay recovery aims to find a recovery policy such that delays in the existing timetables as a result of disruptions are recovered from to minimize a certain objective (e.g., Wang and Meng 2012a, Brouer et al. 2013, Li et al. 2015, and Li et al. 2016 in liner shipping; Corman et al. 2010 and Binder and Albrecht 2012 in public transport; and Rosenberger et al. 2003, Petersen et al. 2012, Aktürk et al. 2014, Maher 2015, and Arikan et al. 2016 in air transport). Note that these studies encompass a wide range of modeling and solution approaches, and that recovery strategies include travel time (or speed) adjustments and rerouting decisions. Visentini et al. (2014) review recovery actions in general transportation, while Psaraftis and Kontovas (2013) provide an overview speed models for energy-efficient maritime transportation. Note that none of these papers study the influence of rearranging the available buffer time in the existing timetables.

\subsection{Robust Planning}

Robust planning constructs timetables that perform well under uncertainty. Wu and Caves (2003) and Wu
(2005) show the importance of buffer time allocation on punctuality in air transport using a simulation approach, and this importance extends to other modes of transportation. Two different approaches are used in literature to construct robust timetables. First, the total available buffer time in an existing schedule can be rearranged to obtain more robust networks (e.g., Kroon, Dekker, and Vromans 2007; Kroon et al. 2008; Fischetti, Salvagnin, and Zanette 2009; Hassannayebi, Sajedinejad, and Mardani 2014; and Wu et al. 2015 in public transport; and Lan, Clarke, and Barnhart 2006; AhmadBeygi, Cohn, and Lapp 2010; and Chiraphadhanakul and Barnhart 2013 in air transport). All of these studies only consider the allocation of buffer times (also framed as time supplements or slack time) in the schedule, but do not consider recovery strategies when disruptions occur. Second, schedules satisfying certain robustness concepts can be constructed. Du, Meng, and Wang (2015) and Norlund, Gribkovskaia, and Laporte (2015) describe methods to design robust schedules that minimize the fuel consumption in shipping taking into account uncertain weather conditions. However, only the fuel consumption of the planned schedule without recovery strategies is taken into account. Cucala et al. (2012) and Duran, Gürel, and Aktürk (2015) consider similar problems for public and air transport, respectively. These papers also determine an optimal speed policy together with the constructed timetable, but the speed is independent of incurred delays.

\subsection{Robust Planning and Delay Recovery}

Robust planning and delay recovery are interrelated, and in recent years there has been increasing interest in approaches for robust planning that incorporates some form of delay recovery during timetable execution. Various approaches to incorporate wait-depart decisions in timetabling exist: a genetic algorithm (Engelhardt-Funke and Kolonko 2004), a light robustness concept for timetabling combined with scenario-based wait-depart decisions (Liebchen et al. 2010), and a recoverable robustness concept that aims to find timetables that are recoverable when disruptions occur (Cicerone et al. 2009, 2012). Furthermore, Gong et al. (2014) develop a two-stage approach based on a genetic algorithm to solve the problem. The first stage considers the timetable optimization and the second stage the speed optimization, in a deterministic setting.

Two-stage stochastic programming (SP) with sample average approximation (SAA) applies naturally to robust timetabling under stochastic delays (Kroon, Dekker, and Vromans 2007; Kroon et al. 2008; Fischetti, Salvagnin, and Zanette 2009), and Qi and Song (2012) and Wang and Meng (2012b) have extended this approach to take into account speed adjustments. In particular, in all of these approaches the timetabling decision (buffer allocation) constitutes the first stage of the SP model, while the second stage expected costs (e.g., delays and possibly 
recovery / fuel costs) are approximated based on a Monte Carlo sample of the underlying random variables-i.e., the delay incurred on individual legs. This entire problem is subsequently formulated and solved as a mixed integer program (MIP). This approach is widely used for stochastic optimization, but the requirement to model the timetable operation as an MIP imposes restrictions on the modeling assumptions that can be accommodated. For example, Qi and Song (2012), who were the first to include speed decisions in timetable construction, make the approximative assumption that delays can (and are) always recovered in a single leg and thus never propagate to the next leg. Wang and Meng (2012b) develop a clever approach that does allow delays to propagate from leg to leg. However, the limitations of the SAA methodology forces them to make a few other assumptions: They must assume that for at least one port in the round tour the arrival delay is always zero. Additionally, they fix so-called target arrival times during timetable development. If possible given the delay, sailing speed must adhere to these targets, and otherwise the ship must sail at maximum speed. This allows the sailing speed to be expressed in the MIP variables, but such speeding decisions typically do not constitute optimal recovery actions (see, e.g., Li et al. 2016). Finally and most importantly, it is important to note that all SP approaches discussed in this paragraph suffer from optimality loss due to the Monte Carlo approximation, and typically from additional optimality loss because the large MIPs cannot be solved to optimality.

\subsection{Our Contribution}

The novelty of our model for timetabling/buffer allocation subject to stochastic delays lies in the use of a stochastic dynamic program (SDP) to model timetable executioni.e., occurrence and propagation of delays and dynamic speed adjustments. The extant literature on stochastic timetabling is based on two-stage stochastic programming and uses Monte Carlo samples to evaluate the timetable. This methodology has inherent limitations. Our contribution is in overcoming these limitations by developing theory and algorithms to efficiently solve our SDP-based timetabling model. Details are given next.

The use of dynamic programs (including SDP) for optimizing speed adjustments in liner shipping was recently pioneered by $\mathrm{Li}, \mathrm{Qi}$, and Lee (2015) and $\mathrm{Li}, \mathrm{Qi}$, and Song (2016), and the operational problem that we consider in this paper is the same as the problem with regular uncertainties in $\mathrm{Li}, \mathrm{Qi}$, and Song (2016). (We emphasize that Li, Qi, and Lee 2015 and $\mathrm{Li}, \mathrm{Qi}$, and Song 2016 do not consider timetabling.) Our theoretical development focuses on analyzing and solving problems that involve decisions on multiple time scales. Specifically, the developed theory deals with the one-time buffer allocation decisions that together specify the timetable, because it is impossible to integrate these decisions into an SDP without losing optimality guarantees. Our key theoretical results are that the costs of operating a timetable with a given buffer allocation are joint convex as a function of that buffer allocation, and that there exists a simple and efficient algorithm for computing subgradients of this function. (Incidentally, we also prove some new results for the operational problem-e.g., conjecture 1 in $\mathrm{Li}, \mathrm{Qi}$, and Song 2016.) These insights underlie the main contribution of this paper: the (to our knowledge) first algorithm for buffer allocation that uses SDP to model timetable execution. This contribution constitutes a range of advantages compared with the state-of-the-art in stochastic timetabling and opens up numerous avenues for further research. We name four key advantages/contributions here. Two contributions arise because our timetabling algorithm models timetable execution as an SDP:

1. We select the optimal online speed during timetable execution (cf. Li, Qi, and Song 2016). Alternatives either assume given (exogenous) speed (Kroon et al. 2008, Fischetti, Salvagnin, and Zanette 2009), or use heuristics to determine the travel speed during timetable operation (Qi and Song 2012, Wang and Meng 2012b).

2. We do not need to make any assumptions that restrict the propagation of delays. Qi and Song (2012) assume that delays never propagate from leg to leg. Wang and Meng (2012b) assume that delays never propagate from round to round, such that Monte Carlo simulation can be performed for each round separately.

By leveraging the structure that we prove for the timetable cost function, our algorithm can directly optimize the exact expected costs. Two more key contributions are related to this:

3. We develop the (to our knowledge) first exact algorithm for timetabling under stochastic delays. Other approaches lose optimality by approximating the objective function using Monte Carlo samples (e.g., Kroon et al. 2008; Fischetti, Salvagnin, and Zanette 2009; Wang and Meng 2012b); see Kroon et al. (2008) for details on the impact of sampling.

4. Our approach avoids solving large-scale MIPs and is therefore very fast: it finds optimal buffers/ timetables in about 70 seconds for realistic problems. For comparison, the computation times of, e.g., Wang and Meng (2012b) and Kroon et al. (2008) are 30-60 minutes and more than one hour, respectively. We can to some extent explain theoretically the superior scalability of our approach-e.g., our results guarantee that evaluating a polynomial number of timetables suffices to find the optimal timetable.

Note that our work focuses on a single ship making round trips (cf. Qi and Song 2012; Wang and Meng 2012b; Li, Qi, and Song 2016). In light of the advantages of our approach, the extensions to railway topologies (cf. Kroon et al. 2008; Fischetti, Salvagnin, and Zanette 2009) and multiple ships (cf. Reinhardt et al. 2016) would 
be very interesting; see Section 7 for a detailed discussion of these and other directions for future research.

\section{The Model}

\subsection{Conceptual Design}

In this section, we first sketch the decision hierarchy in liner shipping and then zoom in to the problem on which we will focus. The decisions on top of the hierarchy are trade selection and fleet design, which are considered to be strategic problems (see, e.g., Christiansen, Fagerholt, and Ronen 2004; Notteboom and Vernimmen 2009; Mulder and Dekker 2018), followed by the liner service design problem that combines strategic and tactical decisions. Ducruet and Notteboom (2012) provide a clear overview of the decisions that are considered in the liner service design problem-namely, the determination of (1) the type of liner service network, (2) the loops (also: routes/round tours) (i.e., how many ports are visited together with the selection of those ports and the determination of the order), (3) the vessel speed, (4) the frequency, and (5) the allocation of vessels.

After the loops have been decided on, liner companies start to negotiate with terminals to make agreements on berthing times, such that they can design a cyclic schedule with arrival and departure times and publish a timetable to their customers. In this paper, we are interested in this latter problem, which arises at the tactical planning level and needs to be solved once every 6-12 months (Mulder and Dekker 2018). At the operational level, ships subsequently encounter delays, and decisions are taken on their actual speed to avoid large deviations from the timetable. These speed decisions can be taken at the start of every leg.

As explained above, the route and ship allocation are already fixed before the problem considered in this paper occurs. Furthermore, the above-mentioned negotiation process with terminals is very complex because the negotiation process with one terminal might influence the negotiations with other terminals. Therefore, it is undesirable to change too many berthing windows at the same time. Hence, we focus on a single route in this study; see also Section 7.

Our method designs reliable timetables at the tactical planning level by dividing the total buffer time over the individual legs. To make optimal timetabling decisions, we consider the consequences of delays incurred at the operational planning level, which is in line with extant literature on timetable optimization in both railway (Kroon, Dekker, and Vromans 2007; Kroon et al. 2008; Fischetti, Salvagnin, and Zanette 2009) and liner shipping (Qi and Song 2012, Wang and Meng 2012b) applications. We consider the total buffer time to be fixed and given in this paper, which corresponds to a fixed number of ships used for the route in our model. Our basic model imposes no restrictions on the allocation of buffers, but Section 5.4 extends the model to include transit time restrictions for the route under consideration. See Section 7 for a discussion and future research agenda on these latter topics.

We now sketch how our model includes the tactical buffer time allocation while taking into account operational delays and recovery actions. In line with the hierarchy sketched above, we consider a round tour with a fixed sequence of port calls and a total planned duration of $T$ time units. We will see that fixing $T$ also fixes the total buffer time $B$. The objective then is to construct an optimal timetable/ schedule by dividing the total buffer time $B$ over the legs of the round tour in such a way that the operating costs for the timetable are minimized. In line with the decision hierarchy, this buffer time allocation decision is taken once (at the tactical level), before timetable operation starts.

To determine the operating costs for a timetable, we let the ship sail a route consisting of $R$ round tours for a planned duration of $R T$. The operating costs while sailing this route consist in the costs of port arrivals and departures that are delayed with respect to the timetable, and the costs of optimally performing recovery actions such as speed adjustments. We do not consider other components of the route costs or administrative costs, as they depend on the chosen route and hence are fixed for our problem. We eventually let $R \rightarrow \infty$ and focus on the long-run average costs, which can be obtained by averaging the total route costs over time. (This limit is convenient from a practical point of view, but not essential to our approach-see Section 7.)

In the remainder of this section, we formally model the tactical and operational level problems. An overview of the necessary notation is given in Table 1.

\subsection{The Tactical Planning Level}

Denote the ports visited in the round tour by $P=$ $\{1, \ldots,|P|\}$. Rounds start in port 1 , visit ports $2,3, \ldots$, $|P|-1,|P|$, and then return to port 1 , after which a new round starts. The route consists of $R$ round tours and $N=R|P|+1$ port calls (including the final port call in port 1). Let $n \in\{1, \ldots, N\}$ index the port calls. The $n$th port call is made at port $p[n]$. Thus, $p[n]:=p$ for $n=p,|P|+p, 2|P|+p, \ldots$, with $p \in P$.

Let $t_{n}^{a r r}$ and $t_{n}^{d e p}$, respectively, denote the planned arrival and departure time of port call $n$. The planned arrival time of port call $n+1$ equals the planned departure time of port call $n$ plus the planned sailing time. This planned sailing time consists of the fixed minimum sailing time needed between ports $p[n]$ and $p[n+1]$ (denoted by $t_{p[n]}^{s}$ ) and the buffer time included in the sea leg, which is a decision variable that will be denoted by $\vec{B}_{p[n]}$. Thus, $t_{n+1}^{a r r}=t_{n}^{d e p}+t_{p[n]}^{s}+\vec{B}_{p[n]}$. The requirement of a cyclic schedule means that buffer time and minimum sailing time for a specific sea leg must be the same for each round. (The notation $t_{p[n]}^{s}$ and $\vec{B}_{p[n]}$ 
Table 1. Overview of the Notation Used in the Paper

\begin{tabular}{|c|c|}
\hline Symbol & Description \\
\hline \multicolumn{2}{|l|}{ Overall model } \\
\hline$T$ & Total planned round tour duration \\
\hline$R$ & Number of round tours in the route \\
\hline$P$ & Set of ports in round tour, indexed by $1,2, \ldots,|P|$ \\
\hline$p, q$ & Port indices \\
\hline$N$ & Number of port calls in the route \\
\hline$n$ & Port call index \\
\hline$p[n]$ & Port at which the $n$th port call is made \\
\hline$d$ & Delay \\
\hline$\overline{\mathscr{B}}$ & Set of feasible buffer vectors \\
\hline $\mathscr{B}$ & 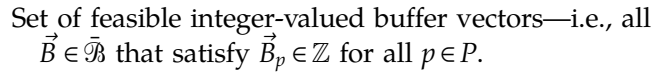 \\
\hline \multicolumn{2}{|c|}{ Tactical planning level } \\
\hline$t_{n}^{a r r}$ & Planned arrival time for port call $n$ \\
\hline$t_{n}^{d e p}$ & Planned departure time from port call $n$ \\
\hline$t_{p}^{s}$ & $\begin{array}{l}\text { Fixed minimum sailing time between port } p \text { and the } \\
\text { next port }\end{array}$ \\
\hline$t_{p}^{p}$ & Fixed port time in port $p$ \\
\hline$\vec{B}$ & $\begin{array}{l}\text { Vector with buffer times: } \vec{B}_{p} \text { denotes the buffer for } \\
\text { port } p\end{array}$ \\
\hline$B$ & Total available buffer time \\
\hline \multicolumn{2}{|c|}{ Operational planning level } \\
\hline$X_{n}^{p}$ & Random delay incurred in port during port call $n$ \\
\hline$X_{n}^{s}$ & Random delay incurred on sea leg after port call $n$ \\
\hline$\tau_{n}$ & Sailing time action on sea leg after port call $n$ \\
\hline$\gamma_{n}$ & Extreme recovery action during port call $n$ \\
\hline$d_{n}^{a r r}$ & Arrival delay for port call $n$ \\
\hline$d_{n}^{d e p}$ & Departure delay from port call $n$ \\
\hline$\tau_{p}^{u}$ & $\begin{array}{l}\text { Upper bound on sailing time action on sea legs after } \\
\text { visiting port } p\end{array}$ \\
\hline $\mathscr{K}_{n}(d ; \vec{B})$ & $\begin{array}{l}\text { Help function at port call } n \text { with delay } d \text { for buffer } \\
\text { allocation } \vec{B} \text {; see (4) }\end{array}$ \\
\hline $\mathscr{T}_{n}(d ; \vec{B})$ & $\begin{array}{l}\text { Optimal sailing time action on sea leg after port call } n \\
\text { with departure delay } d\end{array}$ \\
\hline $\mathscr{Y}_{n}(d ; \vec{B})$ & $\begin{array}{l}\text { Optimal extreme recovery action during port call } n \\
\text { with delay } d\end{array}$ \\
\hline \multicolumn{2}{|l|}{ Cost functions } \\
\hline $\mathscr{D}_{p}^{a r r}(d)$ & Delay cost for arrival with delay $d$ in port $p$ \\
\hline $\mathscr{D}_{p}^{d e p}(d)$ & Delay cost for departure with delay $d$ from port $p$ \\
\hline $\mathscr{F}_{p}(\tau)$ & $\begin{array}{l}\text { Fuel cost for sailing from port } p \text { to the next port using } \\
\text { sailing time action } \tau\end{array}$ \\
\hline$c^{e}$ & Cost per time unit of the extreme recovery action \\
\hline $\mathscr{C}_{n, N}^{a r r}(d ; \vec{B})$ & $\begin{array}{l}\text { Total expected cost for completing the route when } \\
\text { arriving in port call } n \text { with delay } d \text {, assuming } \\
\text { optimal sailing times and extreme recovery actions } \\
\text { for port calls }\{n, \ldots, N\}\end{array}$ \\
\hline $\mathscr{C}_{n, N}^{d e p}(d ; \vec{B})$ & $\begin{array}{l}\text { Total expected cost for completing the route when } \\
\text { departing from port call } n \text { with delay } d \text {, assuming } \\
\text { optimal sailing times and extreme recovery actions } \\
\text { for port calls }\{n, \ldots, N\}\end{array}$ \\
\hline $\mathscr{C}^{*}(\vec{B})$ & $\begin{array}{l}\text { Long-run average optimal costs under buffer } \\
\quad \text { allocation } \vec{B}\end{array}$ \\
\hline $\mathscr{C}^{*}=\mathscr{C}^{*}\left(\vec{B}^{*}\right)$ & $\begin{array}{l}\text { Long-run average optimal costs under an optimal } \\
\text { buffer allocation } \vec{B}^{*}\end{array}$ \\
\hline
\end{tabular}

Note. More detailed explanations can be found in the text. implicitly enforces this requirement; see the definition of $p[n]$.) The planned departure time of the ship for port call $n$ is simply the planned arrival time plus the fixed port time, which will be denoted by $t_{p[n]}^{p}$. Thus, $t_{n}^{\text {dep }}=$ $t_{n}^{a r r}+t_{p[n]}^{p}$. The results in this paper can be extended to optimize buffers for the ports as well, but we do not include such variables because buffer times in ports are expensive and therefore uncommon.

We can set $t_{1}^{d e p}:=0$ without loss of generality. Then, all planned arrival and departure times for the remaining $R|P|$ port calls follow from the above recursive relations once we fix $\vec{B}:=\left(\vec{B}_{1}, \ldots, \vec{B}_{|P|}\right) \in R_{\geq 0}^{|P|}$. So finding a schedule consists in fixing $\vec{B}$. The requirement that the total planned duration equals $T$ implies that $\vec{B}$ should satisfy $\sum_{p \in P} \vec{B}_{p}=B$, where $B:=T-\sum_{p \in P} t_{p}^{p}-$ $\sum_{p \in P} t_{p}^{s}$. (We assume $T \geq \sum_{p \in P} t_{p}^{p}+\sum_{p \in P} t_{p}^{s}$, such that $B \geq 0$.) Since liner ships operate on weekly schedules, the total planned duration $T$ will be an integer multiple of the amount of time units in one week.

\subsection{The Operational Planning Level}

While the ship sails the route, unforeseen events cause the ship to be delayed with respect to the planned timetable-i.e., the planned arrival and departure times $t_{n}^{a r r}$ and $t_{n}^{d e p}$. Discussions at a large liner carrier have revealed that both delays in the port and delays during the sea leg are important (see Wang and Meng 2012a). Therefore, let $X_{n}^{p} \geq 0$ and $X_{n}^{s} \geq 0$ denote the random delay incurred during port call $n$, and in the sea leg after port call $n$, respectively. The random variables $X_{n}^{p}$ and $X_{n}^{s}$ are assumed to be independent of each other, and of all other random variables, in particular of $X_{n^{\prime}}^{p}$ and $X_{n^{\prime}}^{s}$ for $n \neq n^{\prime}$. Independence is reasonable for many causes of delays, but there are exceptions-e.g., a storm could impact both port operations and the ship's voyage out of port. Distributions are arbitrary, but the random delay in a port in a specific position in the round trip is identically distributed in each round trip. Thus, $X_{n}^{p}$ and $X_{n^{\prime}}^{p}$ are identically distributed if $p[n]=p\left[n^{\prime}\right]$. Similarly, $X_{n}^{s}$ and $X_{n^{\prime}}^{s}$ are identically distributed if $p[n]=p\left[n^{\prime}\right]$.

To reduce the delay with respect to the schedule, the liner company can perform two types of recovery actions. Speed adjustments during the sea leg are the preferred approach to deal with delays. But in case of excessive delays, extreme (recovery) actions in the port are sometimes taken in practice, such as cut-and-go. In cut-and-go, the vessel will stop (un)loading and will immediately leave the port. Let $\tau_{n}$ be the difference in the time used to sail from port $p[n]$ to port $p[n+1]$ (excluding unforeseen delays) and the minimum sailing time needed. We will refer to $\tau_{n}$ as the additional sailing time or the sailing time action. Intuitively, increasing $\tau_{n}$ increases the travel time and reduces speed and fuel consumption. Let $\gamma_{n}$ denote the time recovered 
by the extreme recovery action in the $n$th port, which is taken after the port delay is revealed. Note that $\tau_{n}$ and $\gamma_{n}$ are online decision variables, these decisions are taken dynamically in each port and before each sea leg. In contrast, all buffer times $\vec{B}$ are decided on before the ship starts sailing the route.

The following recursive relations for $1 \leq n<N$ govern the propagation of the delay during the trip:

$$
\begin{aligned}
& d_{n+1}^{a r r}=\left(d_{n}^{d e p}+\tau_{n}-\vec{B}_{p[n]}+X_{n}^{s}\right)^{+}, \\
& d_{n}^{\text {dep }}=\left(d_{n}^{a r r}+X_{n}^{p}-\gamma_{n}\right)^{+},
\end{aligned}
$$

where $x^{+}=\max \{x, 0\}$. Since ships have to adhere to the berthing plans made by terminal operators, we assume that ships cannot arrive early in a port. And a ship is not allowed to depart earlier than the schedule, because export containers may arrive just in time to be loaded according to the schedule.

\subsection{Costs}

For $p \in P$, let $\mathscr{D}_{p}^{\text {arr }}(d)$ and $\mathscr{D}_{p}^{d e p}(d)$ be the cost of arriving in and departing, respectively, from port $p$ of the round tour with a delay of $d$ time units with respect to the schedule. We assume that both $\mathscr{D}_{p}^{\text {arr }}(d)$ and $\mathscr{D}_{p}^{d e p}(d)$ are convex and increasing in $d$. Penalizing the average delay satisfies this assumption and is arguably the most intuitive approach for measuring delays. This latter approach is common (e.g., Kroon et al. 2008; Fischetti, Salvagnin, and Zanette 2009), but more general delay cost models have also been proposed (Wang and Meng 2012b).

Let $\mathscr{F}_{p}(\tau)$ denote the fuel cost incurred between port $p$ and the next port when using a sailing time of $t_{p}^{s}+\tau$ time units. $\mathscr{F}_{p}(\tau)$ is decreasing and convex in $\tau$. Indeed, for economic sailing speeds the bunker consumption rate can be accurately approximated by a constant times the third power of sailing speed (Notteboom and Vernimmen 2009, Brouer et al. 2014), which implies that $\mathscr{F}_{p}(\tau)$ is proportional to $1 /\left(t_{p}^{s}+\tau\right)^{2}$, which is decreasing and convex in $\tau$. (For details, see Section 6.1.) Furthermore, let $\tau_{p}^{u} \geq 0$ be the upper bound on the sailing time action obtained from the minimum sailing speed. Then, $\mathscr{F}_{p}(\tau)$ is well defined for all $0 \leq \tau \leq \tau_{p}^{u}$. Denote the costs of using the extreme recovery action to reduce the delay by one unit of time by $c^{e}>0$. This cost is independent of the port for ease of exposition, but it would be straightforward to make it port-dependent.

We next give a stochastic dynamic programming (SDP) formulation of the operational planning level (cf. Bertsekas 2007). Remember that the sailed route consists of $N=R|P|+1$ port calls. Let $\varphi_{n, N}^{\text {arr }}(d ; \vec{B})$ denote the total expected cost for completing the route when arriving for port call $n$ with a delay of $d$ time units, and assuming optimal sailing times and extreme recovery actions for port calls $\{n, \ldots, N\}$. Similarly, let $\mathscr{C}_{n, N}^{d e p}(d ; \vec{B})$ denote these costs when departing from port call $n$. The parameter $\vec{B}$ is added to emphasize that these costs depend on the timetable $\vec{B}$ that is used. The following SDP relation holds for $1 \leq n<N$ :

$$
\begin{aligned}
\mathscr{C}_{n, N}^{d e p}\left(d_{n}^{d e p} ; \vec{B}\right)= & \mathscr{D}_{p[n]}^{d e p}\left(d_{n}^{d e p}\right) \\
& +\min _{0 \leq \tau \leq \tau_{p[n]}^{u}}\left\{\mathscr{F}_{p[n]}(\tau)+\mathscr{K}_{n}\left(d_{n}^{d e p}+\tau ; \vec{B}\right)\right\},
\end{aligned}
$$

where

$$
\begin{aligned}
& \mathscr{K}_{n}\left(d_{n}^{\text {dep }}+\tau ; \vec{B}\right) \\
& \quad:=\mathbb{E}_{X_{n}^{s}}\left[\varphi_{n+1, N}^{\text {arr }}\left(\left(d_{n}^{d e p}+\tau-\vec{B}_{p[n]}+X_{n}^{s}\right)^{+} ; \vec{B}\right)\right] .
\end{aligned}
$$

Note that we select the optimal sailing time action $\tau$. This relation then holds because the total expected costs for completing the trip when departing from port call $n$ with delay $d_{n}^{\text {dep }}$ consist of the departure delay costs $\mathscr{D}_{p[n]}^{d e p}\left(d_{n}^{d e p}\right)$, the sailing costs $\mathscr{F}_{p[n]}(\tau)$, and the optimal expected costs for completing the route when arriving at the next port call with arrival delay $\left(d_{n}^{d e p}+\tau-\vec{B}_{p[n]}+X_{n}^{s}\right)^{+}$(see (1)). By definition of $\mathscr{C}_{n+1, N}^{a r r}$, those latter costs equal $\mathscr{C}_{n+1, N}^{\text {arr }}\left(\left(d_{n}^{d e p}+\tau-\vec{B}_{p[n]}+X_{n}^{s}\right)^{+} ; \vec{B}\right)$. We take the expectation to account for the randomness of $X_{n}^{s}$. Similarly, the following SDP relation holds for $1<n<N$ :

$$
\begin{aligned}
& \mathscr{C}_{n, N}^{\text {arr }}\left(d_{n}^{\text {arr }} ; \vec{B}\right) \\
& =\mathscr{D}_{p[n]}^{\text {arr }}\left(d_{n}^{\text {arr }}\right)+\mathbb{E}_{X_{n}^{p}}\left[\min _{\gamma \geq 0}\left\{c^{e} \gamma+\mathscr{C}_{n, N}^{\text {dep }}\left(\left(d_{n}^{\text {arr }}+X_{n}^{p}-\gamma\right)^{+} ; \vec{B}\right)\right\}\right] .
\end{aligned}
$$

Note that here we use (2), and that the extreme recovery action is taken after the port delay is incurred. For the final arrival in port 1 , we have the following:

$$
\mathscr{C}_{N, N}^{a r r}\left(d_{N}^{\text {arr }} ; \vec{B}\right)=\mathscr{D}_{p[N]}^{\text {arr }}\left(d_{N}^{\text {arr }}\right) .
$$

Therefore, for the final arrival, we only pay the delay costs.

We introduce notation regarding the optimal sailing times and extreme recovery actions. Let $\mathscr{T}_{n}(d ; \vec{B})$ denote the optimal sailing time after port call $n$ (on the sea leg towards port call $n+1$ ) when the departure delay equals $d$ :

$$
\begin{gathered}
\mathscr{T}_{n}(d ; \vec{B}):=\min \left\{\tau^{\prime} \mid \tau^{\prime} \in \underset{0 \leq \tau \leq \tau_{p[n]}^{u}}{\arg \min }\left\{\mathscr{F}_{p[n]}(\tau)\right.\right. \\
\left.\left.+\mathscr{K}_{n}(d+\tau ; \vec{B})\right\}\right\} .
\end{gathered}
$$


Let $\mathscr{Y}_{n}\left(d+X_{n}^{p} ; \vec{B}\right)$ denote the optimal extreme recovery action in port call $n$ when the delay (including port delay) equals $d+X_{n}^{p}$ :

$$
\begin{aligned}
& \mathscr{Y}_{n}\left(d+X_{n}^{p} ; \vec{B}\right) \\
& :=\max \left\{\gamma^{\prime} \mid \gamma^{\prime} \in \underset{\gamma \geq 0}{\arg \min }\left\{c^{e} \gamma+\mathscr{C}_{n, N}^{\text {dep }}\left(\left(d+X_{n}^{p}-\gamma\right)^{+} ; \vec{B}\right)\right\}\right\} .
\end{aligned}
$$

So as a tie-breaking rule, we use minimization of the delay in the next port.

\subsection{The Long-Run Average Costs}

Because the buffers are transformed into a timetable that is operated for many rounds, we adopt the longrun average costs as performance criterion, which will be denoted by $\mathscr{C}^{*}(\vec{B})$ and is defined as follows:

$$
\mathscr{C}^{*}(\vec{B}):=\lim _{R \rightarrow \infty} \frac{\mathscr{C}_{1, R|P|+1}^{d e p}\left(d_{1}^{d e p} ; \vec{B}\right)}{R}, \quad \forall \vec{B} \in \overline{\mathscr{B}},
$$

where $\overline{\mathscr{B}}=\left\{\vec{B} \in \mathbb{R}_{\geq 0}^{|P|} \mid \sum_{p \in P} \vec{B}_{p}=B\right\}$. Intuitively, the limit on the RHS of (9) exists and is independent of $d_{1}^{\text {dep }}$, and Theorem 1 formally establishes this result under appropriate conditions. This paper then studies the following optimization problem:

$$
\mathscr{C}^{*}=\min _{\vec{B} \in \overline{\mathscr{P}}} \mathscr{C}^{*}(\vec{B}) .
$$

This problem is nonstandard. Each buffer allocation $\vec{B} \in \overline{\mathscr{B}}$ yields an SDP (governed by (3)-(6)) whose optimal long-term average costs equals $\mathscr{C}^{*}(\vec{B})$. But the buffer time variables themselves cannot be accommodated for in the SDP because they are one-time decisions that affect multiple states: after each departure from port $p$, the arrival delay in the next port is affected by $\vec{B}_{p}$. Note that the sailing speed decisions are part of the SDP, so the problem optimizes the buffer allocation taking into account sailing speed optimization during timetable execution.

\subsection{Assumptions for Computational Purposes}

In general, solving for the optimal costs $\mathscr{C}^{*}(\vec{B})$ of the SDP that arises for fixed buffers $\vec{B}$ is already computationally intractable. This is because the SDP has a continuous state space because delay is continuous. (Apart from the current delay, the state consists of the current port $p[n]$ and whether we are arriving or departing.) To deal with this computational issue, we will assume discrete delays and piecewise linear fuel costs (see also Wang and Meng 2012a, Reinhardt et al. 2016), who use similar discretization assumptions). Specifically, after an appropriate basic time unit is chosen (for example, the time unit in which timetables are communicated), we assume the following.
Assumption 1 (Discrete Model Primitives). The delays $X^{s}$ and $X^{p}$ take on values in $\mathbb{Z}_{\geq 0}$. The total buffer $B$ and the maximum additional sailing time $\tau_{p}^{u}$ are in $\mathbb{Z}_{\geq 0}$. The functions $\mathscr{D}_{p}^{\text {arr }}(\cdot), \mathscr{D}_{p}^{\text {dep }}(\cdot)$, and $\mathscr{F}_{p}(\cdot)$ are piecewise linear functions, with breakpoints on $\mathbb{Z}_{\geq 0}$. The initial delay is in $\mathbb{Z}_{\geq 0}$.

We now discuss another computational issue. If we encounter large sea and port delays repeatedly, the delay with respect to the schedule may grow arbitrarily large. In practice, it seems reasonable to assume that when delay exceeds some (possibly large) threshold, it will be optimal to perform the extreme recovery action. We therefore make this assumption to simply and straightforwardly bound the maximum delay. For ease of exposition, we will also assume that the random sea and port delays are bounded by some arbitrary number. These assumptions will simplify the computation of the optimal costs associated with a buffer $\vec{B} \in \overline{\mathscr{B}}$.

Assumption 2 (Bounded Delays). For each $p \in P$, there exists a delay $d_{p}^{\max }<\infty$ such that $\mathscr{D}_{p}^{\text {dep }}(d)-c^{e} d$ is monotonically increasing $\forall d>d_{p}^{\max }$. There exist $X_{p}^{s, \max }$, $X_{p}^{p, \max } \in \mathbb{Z}_{\geq 0}$ such that $\forall n: \mathbb{P}\left(X_{n}^{s}>X_{p[n]}^{s, \max }\right)=0, \mathbb{P}\left(X_{n}^{p}>\right.$ $\left.X_{p[n]}^{p, \max }\right)=0$.

These assumptions are not restrictive in practice as $d_{p}^{\max }, X_{p}^{\text {s,max }}$, and $X_{p}^{\mathrm{p}, \max }$ can be taken to be large (e.g., one or more weeks when operating a weekly schedule).

\section{Theoretical Insights}

In this section, we derive various theoretical insights into the problem. The proofs are in Online Appendix A. We first present four lemmas that provide insight in optimal recovery actions and buffer allocations. These results can be established without Assumptions 1 and 2. The first result verifies that the costs for completing the schedule increase with the present delay.

Lemma 1. The functions $\mathscr{C}_{n, N}^{\text {dep }}(d ; \vec{B})$ and $\mathscr{C}_{n+1, N}^{\text {arr }}(d ; \vec{B})$ are nondecreasing in the amount of delay $d$ for $1 \leq n<N$ and $\vec{B} \in \overline{\mathscr{B}}$.

The following result is more surprising, since the costs are not separable because delays may propagate from port call to port call.

Lemma 2. The functions $\mathscr{C}_{n, N}^{d e p}(d ; \vec{B})$ and $\varphi_{n+1, N}^{\text {arr }}(d ; \vec{B})$ are joint convex in $\vec{d}$ and $\vec{B} \in \overline{\mathscr{B}}$ for $1 \leq n<N$.

We next give results that shed light onto how the sailing times and extreme actions should depend on the current delay. The following lemma shows that the larger the delay, the more action should be taken. The lemma proves conjecture 1 in $\mathrm{Li}, \mathrm{Qi}$, and Song (2016).

Lemma 3. (a) The optimal sailing time action $\mathscr{T}_{n}\left(d_{n}^{\text {dep }} ; \vec{B}\right)$ between two ports is nonincreasing in the departure delay 
$d_{n}^{\text {dep }}$ for $1 \leq n<N$ and $\vec{B} \in \overline{\mathscr{B}}$; (b) the optimal extreme recovery action $\mathscr{Y}_{n}\left(d_{n}^{\text {arr }}+X_{n}^{p} ; \vec{B}\right)$ in a port is nondecreasing in the amount of delay $d_{n}^{\text {arr }}+X_{n}^{p}$ before that action for $1<n<N$ and $\vec{B} \in \overline{\mathscr{B}}$.

Thus, a ship should increase sailing speed when it has a larger departure delay. To understand the intuition behind the next result, consider a ship that plans to depart at Monday at 1400 hours, and suppose the optimal speed for this departure time implies a planned travel time of 48 hours-i.e., planned arrival Wednesday at 1400 hours. Now suppose an event causes the planned departure to be delayed to Monday at 1800 hours. Without speed increase the planned arrival becomes Wednesday at 1800 hours. But by Lemma 3 the speed should increase and the planned arrival should lie before Wednesday at 1800 hours. But could the optimal planned arrival time lie before 1400 hours? That is, could the optimal speed increase be such that it more than compensates for the delay? The following lemma shows that this can never be the case. Thus, in our example the planned arrival time after incurring the delay must lie between 1400 hours (our original planned arrival time before the delay was incurred) and 1800 hours (the planned arrival time taking into account the delay, but without changing the speed). A similar result holds for extreme actions in the port. These results can be used to more efficiently solve the operational problem.

Lemma 4. (a) The optimal arrival delay $d_{n+1}^{\text {arr }}=\left(d_{n}^{\text {dep }}+\right.$ $\left.\mathscr{T}_{n}\left(d_{n}^{\text {dep }} ; \vec{B}\right)-\vec{B}_{p[n]}+X_{n}^{s}\right)^{+}$is stochastically nondecreasing in the departure delay $d_{n}^{\text {dep }}$, for $1 \leq n<N$ and $\vec{B} \in \overline{\mathscr{B}}$; (b) the optimal departure delay $d_{n}^{\text {dep }}=\left(d_{n}^{\text {arr }}+X_{n}^{p}-\mathscr{Y}_{n}\left(d_{n}^{\text {arr }}+\right.\right.$ $\left.\left.X_{n}^{p} ; \vec{B}\right)\right)^{+}$is nondecreasing in the delay $d_{n}^{\text {arr }}+X_{n}^{p}$ after incurring port delay, for $1<n<N$ and $\vec{B} \in \overline{\mathscr{B}}$.

The last result in this section establishes the joint convexity of $\mathscr{C}^{*}(\vec{B})$ in the decision variables $\vec{B}$. This result is the basis for our solution algorithm, because it implies the existence of subgradients. This theorem requires Assumptions 1 and 2 to ensure that the longrun average $\operatorname{costs} \mathscr{C}^{*}(\vec{B})$ are well defined, which clearly is required to establish their convexity.

Theorem 1. Under Assumptions 1 and 2, the optimal longterm average cost $\lim _{R \rightarrow \infty} \mathscr{C}_{1, R|P|+1}^{\text {dep }}\left(d_{1}^{\text {dep }} ; \vec{B}\right) / R$ exists for all $\vec{B} \in \overline{\mathscr{B}}$, and it is independent of $d_{1}^{\text {dep }}$. Moreover, $\mathscr{C}^{*}(\vec{B})$ is joint convex in $\vec{B} \in \overline{\mathscr{P}}$.

This result will be used in the next section to find the optimal buffer $\vec{B}^{*}$.

\section{Solution Approach}

We develop an algorithm for finding a $\vec{B} \in \overline{\mathscr{P}}$ that minimizes $\mathscr{C}^{*}(\vec{B})$. We make heavy use of Theorem 1 , which guarantees the existence of subgradients of $\mathscr{C}^{*}(\vec{B})$ for arbitrary $\vec{B} \in \overline{\mathscr{B}}$. The novelty of our algorithm lies in developing an approach for efficiently computing these subgradients. We describe this approach in Section 5.1. Section 5.2 develops a subgradient-based algorithm for minimizing $\mathscr{C}^{*}(\vec{B})$. Section 5.3 discusses the optimality and efficiency of this algorithm, and Section 5.4 discusses how to extend it to include transit time requirements.

\subsection{Subgradients}

Theorem 1 implies that $\mathscr{C}^{*}(\vec{B})$ is convex. Thus, for each $\vec{B} \in \overline{\mathscr{B}}$, there exists a subgradient (see, e.g., Nesterov 2013). In our setting, a subgradient of $\mathscr{C}^{*}(\vec{B})$ at $\vec{B}$ is defined as a vector $g=\left(g_{1}, \ldots, g_{|P|}\right)$ that satisfies

$$
\forall \vec{B}^{\prime} \in \overline{\mathscr{B}}: \mathscr{C}^{*}\left(\vec{B}^{\prime}\right) \geq \mathscr{C}^{*}(\vec{B})+\sum_{p \in P} g_{p}\left(\vec{B}_{p}^{\prime}-\vec{B}_{p}\right)
$$

In general, computing subgradients involves analyzing the change of the objective function when the input changes. But changing $\vec{B}$ will also change the speed actions that are optimal, cascading many other changes. Thus, computing subgradients becomes a nontrivial matter. In Online Appendix B we conduct a detailed theoretical analysis of the specialized model that is obtained by imposing discrete model primitives (Assumption 1) and bounded delays (Assumption 2). This analysis yields the algorithm for computing subgradients that is presented next.

Intuitively, to compute the subgradient of $\mathscr{C}^{*}(\vec{B})$ at some $\vec{B}$, we determine $|P|$ integer buffers $\vec{B}^{q}$ for all $q \in P$. These integer buffers must be carefully constructed based on $\vec{B}$. We next determine the costs $\mathscr{C}^{*}(\vec{B} q)$ for each of those buffers. Then the costs $\mathscr{C}^{*}(\vec{B})$, as well as the subgradient at $\vec{B}$, can be found by constructing a hyperplane that interpolates the costs between the points $\overrightarrow{B^{q}}$. Under the assumptions of our model, we can show that this approach gives exact results. We next give the details.

The construction of $\vec{B}^{q}$ involves transforming the buffers to cumulative buffers, and vice versa. Each $\vec{B} \in \overline{\mathscr{B}}$ corresponds to a cumulative buffer allocation $\tilde{B}$, by setting $\tilde{B}_{p}:=\sum_{p^{\prime}=1}^{p-1} \vec{B}_{p^{\prime}}$ and $\tilde{B}=\left(\tilde{B}_{1}, \ldots, \tilde{B}_{|P|}\right)$. (Thus, $\tilde{B}_{1}:=0$.) Note that the regular buffer $\vec{B}$ can be obtained from the cumulative buffer $\tilde{B}$ by setting $\vec{B}_{p}=\tilde{B}_{p+1}-\tilde{B}_{p}$ for $p \in\{1, \ldots,|P|-1\}$, and $\vec{B}_{|P|}=B-\tilde{B}_{|P|}$.

Based on this, Algorithm 1 specifies how to compute a subgradient for our model.

Algorithm 1 (Computing a subgradient $g$ and the costs $\mathscr{C}^{*}(\vec{B})$ at $\left.\vec{B} \in \overline{\mathscr{B}}\right)$

1. Let $\tilde{B} \in \tilde{\mathscr{P}}$ be the cumulative buffer corresponding to $\vec{B} \in \bar{\Re}$.

2. (This step orders the ports based on the fractional parts of $\tilde{B}$.) For all $p \in P$, write $\tilde{B}_{p}=z_{p}+x_{p}$, with $z_{p} \in\{0,1, \ldots, B-1\}$ and $0 \leq x_{p} \leq 1$. (If there are multiple options, select the one with largest $z_{p}$.) 
Let $f: P \rightarrow P$ be the unique permutation of $P$ such that $\forall p, p^{\prime} \in P$ with $p<p^{\prime}$ it holds that either (a) $x_{f(p)}<x_{f\left(p^{\prime}\right)}$ or $(\mathrm{b}) x_{f(p)}=x_{f\left(p^{\prime}\right)}$ and $f(p)<f\left(p^{\prime}\right)$.

3. For each $q \in P$, define the integer cumulative buffer $\tilde{B}^{q}=\left(\tilde{B}_{1}^{q}, \ldots, \tilde{B}_{|P|}^{q}\right) \in \tilde{\mathscr{B}}$ as follows:

$$
\tilde{B}_{p}^{q}= \begin{cases}z_{p} & \text { if } f^{-1}(p) \leq q \\ z_{p}+1 & \text { if } f^{-1}(p)>q .\end{cases}
$$

4. For each $q \in P$, obtain the regular buffer $\vec{B}^{q}$ from the cumulative buffer $\tilde{B}_{\rightarrow}^{q}$ by setting $\vec{B}_{p}^{q}=\tilde{B}_{p+1}^{q}-\tilde{B}_{p}^{q}$ for $p \in\{1, \ldots,|P|-1\}$, and $\vec{B}_{|P|}^{q}=B-\tilde{B}_{|P|}^{q}$.

5. Compute $\mathscr{C}^{*}\left(\vec{B}^{q}\right)$ for each $q \in P$. It corresponds to the long-run average costs of the SDP described in (3)-(6), which can be obtained efficiently using policy iteration or linear programming because all buffers in $\vec{B}^{q}$ are integer-i.e., $\vec{B}^{q} \in \mathscr{B}$ (see Section 5.3).

6. Solve the below system of equations subject to $g_{1}=0$ to obtain $\mathscr{C}^{*}(\vec{B})$ as well as a subgradient $g=\left(g_{1}, \ldots, g_{|P|}\right)$ at $\vec{B}$ :

$$
\mathscr{C}^{*}\left(\vec{B}^{q}\right)=\mathscr{C}^{*}(\vec{B})+\sum_{p \in P} g_{p}\left(\vec{B}_{p}^{q}-\vec{B}_{p}\right), \quad q \in P .
$$

Section 5.3 establishes the exactness of the algorithm. We illustrate the algorithm by example:

Example. Let $B=20,|P|=4$, and thus $P=\{1,2,3,4\}$. Suppose $\vec{B}=(4.6,5.5,2.0,7.9)$ :

1. We obtain $\tilde{B}=\left(\tilde{B}_{1}, \tilde{B}_{2}, \tilde{B}_{3}, \tilde{B}_{4}\right)=(0,4.6,4.6+5.5$, $4.6+5.5+2.0)=(0,4.6,10.1,12.1)$.

2. We find $z_{1}=0, z_{2}=4, z_{3}=10, z_{4}=12$, and $x_{1}=0$, $x_{2}=0.6, x_{3}=0.1, x_{4}=0.1$. From these values we conclude that $f$ must satisfy $x_{f(1)}<x_{f(2)}=x_{f(3)}<x_{f(4)}$. This implies $f(1)=1$ and $f(4)=2$. Since $x_{3}=x_{4}$, we apply condition $\mathrm{b}$ and find $f(2)=3$ and $f(3)=4$.

3. We invert $f$ to obtain $f^{-1}(1)=1, f^{-1}(2)=4$, $f^{-1}(3)=2$, and $f^{-1}(4)=3$. We find: $\tilde{B}^{1}=(0,5,11,13)$, $\tilde{B}^{2}=(0,5,10,13), \tilde{B}^{3}=(0,5,10,12), \tilde{B}^{4}=(0,4,10,12)$.

4. We find: $\vec{B}^{1}=(5,6,2,7), \vec{B}^{2}=(5,5,3,7), \vec{B}^{3}=$ $(5,5,2,8), \vec{B}^{4}=(4,6,2,8)$.

5 . For the sake of the example, suppose the policy iteration yields the following costs: $\mathscr{C}^{*}\left(\vec{B}^{1}\right)=120$, $\mathscr{C}^{*}\left(\vec{B}^{2}\right)=130, \mathscr{C}^{*}\left(\vec{B}^{3}\right)=110, \mathscr{C}^{*}\left(\vec{B}^{4}\right)=145$.

6. We solve the following system of equations subject to $g_{1}=0: \quad 120=\mathscr{C}^{*}(\vec{B})+0.4 g_{1}+0.5 g_{2}+0 g_{3}-$ $0.9 g_{4} ; 130=\mathscr{C}^{*}(\vec{B})+0.4 g_{1}-0.5 g_{2}+1 g_{3}-0.9 g_{4} ; 110=$ $\mathscr{C}^{*}(\vec{B})+0.4 g_{1}-0.5 g_{2}+0 g_{3}+0.1 g_{4} ; 145=\mathscr{C}^{*}(\vec{B})-0.6 g_{1}+$ $0.5 g_{2}+0 g_{3}+0.1 g_{4}$

We solve for the unknowns to obtain: $g_{2}=35, g_{3}=$ $45, g_{4}=25$ and $\mathscr{C}^{*}(\vec{B})=125$.

\subsection{Subgradient-Based Algorithm}

We next develop an efficient algorithm for minimizing $\mathscr{C}^{*}(\vec{B})$ over $\vec{B} \in \mathscr{\Re}$. The algorithm iteratively computes subgradients using Algorithm 1. For the $i$ th iteration, the subgradient $g^{i}$ at $\vec{B}^{i}$ is computed. Denote it by $g^{i}=\left(g_{1}^{i}, \ldots, g_{|P|}^{i}\right)$. Also, $\mathscr{C}^{*}\left(\vec{B}^{i}\right)$ is computed, and we let $g_{0}^{i}=\mathscr{C}^{*}\left(\vec{B}^{i}\right)-\sum_{p \in P} g_{p}^{i} \vec{B}_{p}^{i}$. Then (11) implies that

$$
\forall \vec{B}^{\prime} \in \overline{\mathscr{P}}: \mathscr{C}^{*}\left(\vec{B}^{\prime}\right) \geq \sum_{p \in P} g_{p}^{i} \vec{B}_{p}+g_{0}^{i} .
$$

For iteration 1, we compute the subgradient $g^{1}$ at some (arbitrary) $\vec{B}^{1} \in \overline{\mathscr{B}}$. For iteration $i+1$, we have available $g^{j}$ for $j=1, \ldots, i$, and we determine $\vec{B}^{i+1}$ using the following problem:

$$
\begin{aligned}
& \min z \\
& \text { s.t. } \quad z \geq \sum_{p \in P} g_{p}^{j} \vec{B}_{p}+g_{0}^{j} \quad j \in\{1, \ldots, i\} \\
& \sum_{p \in P} \vec{B}_{p}=B \\
& \vec{B}_{p} \geq 0 \quad p \in P .
\end{aligned}
$$

Denote the optimal solution of (14)-(17) by $\left(z^{\prime}, \vec{B}^{\prime}\right)$. Note that (15) ensures that $z^{\prime}$ satisfies the inequalities (13) imposed by the subgradients. Also, (16) and (17) ensure that $\vec{B}^{\prime} \in \overline{\mathscr{B}}$. Remember that $\mathscr{C}^{*}=\mathscr{C}^{*}\left(\vec{B}^{*}\right)$, where $\vec{B}^{*}$ is an optimal buffer allocation. By (13) we find that $\mathscr{C}^{*}\left(\vec{B}^{*}\right) \geq$ $\sum_{\vec{B}} p \in P g_{p}^{j} \vec{B}_{p}^{*}+g_{0}^{j}$ for $j \in\{1, \ldots, i\}$. Thus, setting $z=\mathscr{C}^{*}$ and $\vec{B}=\vec{B}^{*}$ always gives a feasible solution to (14)-(17). This implies that the optimal solution $\left(z^{\prime}, \vec{B}^{\prime}\right)$ must always satisfy $z^{\prime} \leq \mathscr{C}^{*}$.

In addition, each computation of Algorithm 1 yields upper bounds to $\mathscr{C}^{*}$. In particular, Step $5 \mathrm{com}$ putes $\mathscr{C}^{*}\left(\vec{B}^{q}\right)$ for $q \in P$, and clearly $\mathscr{C}^{*}\left(\vec{B}^{q}\right) \geq \mathscr{C}^{*}$. Let $q^{\prime} \in \arg \min _{q \in P} \mathscr{C}^{*}\left(\vec{B}^{q}\right)$ be the index for the tightest of the upper bounds found in Step 5. Then for each iteration of our optimization algorithm, we obtain the upper bound $\mathscr{C}^{*}\left(\vec{B}^{q^{\prime}}\right)$ and corresponding solution $\vec{B}^{q}$ as part of computing the gradient, and a lower bound when subsequently optimizing (14)-(17). Details are given in Algorithm 2.

\section{Algorithm 2 (Solution algorithm for finding $\vec{B}^{*}$ )}

1. Initialize $i \leftarrow 1, \vec{B} 1=\left(\vec{B}_{1}^{1}, \ldots, \vec{B}_{|P|}^{1}\right)$ with $\vec{B}_{p}^{1}=B /|P|$. Set $U B \leftarrow \infty$ and $L B \leftarrow-\infty$.

2. Execute Algorithm 1: (1) obtain a subgradient $g^{i}$ at $\vec{B}^{i}$, and (2) obtain $\mathscr{C}^{*}\left(\vec{B}^{q}\right)$ for $g \in P$.

3. Let $g^{\prime} \in \operatorname{argmin}_{q \in P} \mathscr{C}^{*}\left(\vec{B}^{q}\right)$. If $\mathscr{C}^{*}\left(\vec{B}^{q^{\prime}}\right)<U B$, set $U B \leftarrow \mathscr{C}^{*}\left(\vec{B} q^{\prime}\right)$ and $\vec{B} U B \stackrel{q}{\leftarrow \in P q^{\prime}}$.

4. Let $\left(z^{\prime}, \vec{B}^{\prime}\right)$ denote the optimal solution of (14)(17). Set $L B \leftarrow z^{\prime}, \vec{B}^{i+1} \leftarrow \vec{B}^{\prime}$.

5. If $U B=L B$, let $\vec{B}^{*} \leftarrow \vec{B}^{U B}$ and terminate. Otherwise, set $i \leftarrow i+1$ and go to Step 2 .

In initial steps, the $\vec{B}^{i+1}$ from Step 4 of Algorithm 2 may lie far away from the last search point $\vec{B}^{i}$, adversely impacting performance. Therefore, we limit 
the distance between $\vec{B}^{i}$ and $\vec{B}^{i+1}=\vec{B}$. Consider the constraints,

$$
\sum_{p \in P}\left|\vec{B}_{p}-\vec{B}_{p}^{i}\right| \leq w^{\max }, \quad \forall p \in P:\left|\vec{B}_{p}-\vec{B}_{p}^{i}\right| \leq w_{p}^{\max } .
$$

Then Step 4 is replaced by the following in the first $I$ iterations of the algorithm:

$4^{\prime}$. Let $\left(z^{\prime}, \overrightarrow{B^{\prime}}\right)$ denote the optimal solution of (14)$(17)+(18)$. Set $\vec{B}^{i+1} \leftarrow \vec{B}^{\prime}$. Let $\left(z^{\prime \prime}, \vec{B}^{\prime \prime}\right)$ denote the optimal solution of (14)-(17). Set $L B \leftarrow z^{\prime \prime}$.

Here, $w^{\max }>0, \forall p \in P: w_{p}^{\max }>0$, and $I \in \mathbb{Z}_{\geq 0}$ are parameters of the algorithm.

\subsection{Theoretical Analysis}

In this section, we prove correctness of Algorithms 1 and 2, and discuss their efficiency. Denote the set of integer buffer vectors by $\mathscr{B}=\left\{\vec{B} \in \mathbb{Z}_{\geq 0}^{|P|} \mid \sum_{p \in P} \vec{B}_{p}=B\right\}$. Note that $\mathscr{B} \subseteq \overline{\mathscr{B}}$. The approach developed in Sections 5.1 and 5.2 rests on Theorem 1 and the following result:

Theorem 2. For any $\vec{B} \in \overline{\mathscr{B}}$, let $\vec{B}^{q}$ for $q \in P$ be the buffers obtained in Algorithm 1, Step 4:

- For $q \in P$, we have $\vec{B}^{q} \in \mathscr{B}$, and $\vec{B}$ is in the convex hull of $\left\{\vec{B}^{q} \mid q \in P\right\}$.

- There exists a subgradient $g=\left(g_{1}, \ldots, g_{|P|}\right)$ at $\vec{B}$, such that $g_{1}=0$ and

$$
\mathscr{C}^{*}\left(\vec{B}^{q}\right)=\mathscr{C}^{*}(\vec{B})+\sum_{p \in P} g_{p}\left(\vec{B}_{p}^{q}-\vec{B}_{p}\right), \quad q \in P .
$$

Formally proving this result requires defining additional concepts and notation. We do so in Online Appendix B, which also contains proofs of the other results in this section. Theorem 2 directly implies that Algorithm 1 yields correct subgradients. Another consequence is the following:

Corollary 1. In finitely many iterations, Algorithm 2 returns an optimal solution $\vec{B}^{*}$ that is integral-i.e., $\vec{B}^{*} \in$ $\arg \min _{\vec{B} \in \overline{\mathscr{P}}} \mathscr{C}^{*}(\vec{B})$ and $\vec{B}^{*} \in \mathscr{B}$.

This result shows that Algorithm 2 is exact. The result also implies there must exist an optimal buffer allocation that is integral-i.e., $\min _{\vec{B} \in \overline{\mathscr{B}}} \mathscr{C}^{*}(\vec{B})=\min _{\vec{B} \in \mathscr{B}} \mathscr{C}^{*}(\vec{B})$.

This is convenient because the timetable communicated to customers typically needs to be integral.

We next discuss the computational complexity of our algorithms. We first analyze the computation time of Algorithm 1. It is dominated by Step 5: the computation of $\mathscr{C}^{*}\left(\vec{B}^{q}\right)$, which corresponds to the long-term average cost of the SDP generated by $\vec{B}^{q}$. The state space of this SDP includes the delay with respect to the model, and delay is continuous in our model. To avoid a continuous state space, we next show that for integer buffers, any fractional actions are dominated and thus the delay always remains integer. Moreover, by the upper bounds $d_{p}^{\max }, X_{p}^{\mathrm{s}, \max }$, and $X_{p}^{p, \max }$ that hold by Assumption 2, we can bound the maximal delay:

Corollary 2. Let $\vec{B} \in \mathscr{B}$, and choose actions $\tau_{n}$ and $\gamma_{n}$ optimally using (7) and (8). Then $\tau_{n} \in \mathbb{Z}_{\geq 0}, \quad \gamma_{n} \in \mathbb{Z}_{\geq 0}$, $d_{n}^{d e p} \in\left\{0, \ldots, d_{p[n]}^{\max }\right\}$, and $d_{n+1}^{a r r}+X_{n+1}^{p} \in\left\{0, \ldots, d_{p[n]}^{\max }+\tau_{p[n]}^{u}+\right.$ $\left.X_{p[n]}^{s, \max }+X_{p[n+1]}^{p, \max }\right\}$.

The SDP associated with integral buffers thus has a finite state space. There are departure states and port states. Departure states $d_{p}^{d e p}=d$ correspond to departing from port $p$ with delay $d$. Port states $d_{p}^{\text {arr }}+$ $X^{p}=d$ correspond to being in port $p$ with a delay $d$, after incurring port delay. Transition probabilities follow from Equations (1) and (2). In particular, when in state $d_{p}^{a r r}+X^{p}=d$ and taking (extreme) action $\gamma$, we transition to state $d_{p}^{d e p}=d^{\prime}$ where $d^{\prime}=(d-\gamma)^{+}$. When in state $d_{p}^{d e p}=d$ and taking (sailing time) action $\tau$, we go to state $d_{p^{\prime}}^{\text {arr }}+X^{p}=d^{\prime}$. Here, $p^{\prime}$ is the port after $p$ in the round tour, and $d^{\prime}=\left(d+\tau-\vec{B}_{p}+X^{s}\right)^{+}+X^{p}$. Transition probabilities then follow from the distribution of $X^{s}$ and $X^{p}$. From Corollary 2, our SDP has $\sum_{p \in P}\left(2+2 d_{p}^{\max }+\tau_{p}^{u}+X_{p}^{\mathrm{s}, \max }+X_{p}^{\mathrm{p}, \max }\right)$ states. To compute $\mathscr{C}^{*}\left(\vec{B}^{q}\right)$, we can use an algorithm for finding the long-run average costs of an SDP that satisfies weak accessibility (cf. Bertsekas 2007). In particular, the LPbased solution approach yields an optimal solution (up to machine precision) in polynomial time in the number of states of our SDP.

We next analyze the number of subgradient computations required to find $\vec{B}^{*}$. Since we optimize the convex function $\mathscr{C}^{*}(\vec{B})$ over the convex bounded domain $\overline{\mathscr{B}}$, and since the optimal solution is integral, the ellipsoid method (Khachiyan 1980) could replace Algorithm 2. This algorithm guarantees iterations (and thus evaluations of $\left.\mathscr{C}^{*}(\vec{B})\right)$ that are polynomial in $|P|$. Algorithm 2 is without such guarantees but may be superior in practice: It is much easier to implement and requires only $17-44$ gradients to find $\vec{B}^{*}$ for a range of instances with 14 ports (see Section 6).

\subsection{Extension: Transit Time Requirements}

To illustrate the flexibility of our approach, we show here how to (crudely) model transit time requirements. Such requirements arise because the transit time is considered by customers to be an important factor when choosing a carrier (Brouer et al. 2014), and liners take this into account when developing the timetable (Reinhardt et al. 2016).

Suppose that the maximal (planned) transit time between ports $p$ and $q$ in $P$ is $T_{\operatorname{tr}}(p, q) \in \mathbb{Z}_{\geq 0}$. Let $V_{p}:=\sum_{p^{\prime}=1}^{p-1}\left(\vec{B}_{p^{\prime}}+t_{p^{\prime}}^{s}+t_{p^{\prime}}^{p}\right)$ be the first planned arrival at port $p \in P$. We plan to visit $p$ at $V_{p}, V_{p}+T, \ldots$, and $q$ at 
$V_{q}, V_{q}+T, \ldots$ We thus model the transit time requirement as

$$
\begin{aligned}
& V_{q}-V_{p} \leq T_{\operatorname{tr}}(p, q) \Leftrightarrow \\
& \sum_{p^{\prime}=p}^{q-1} \vec{B}_{p^{\prime}} \leq T_{\operatorname{tr}}(p, q)-\sum_{p^{\prime}=p}^{q-1}\left(t_{p^{\prime}}^{s}+t_{p^{\prime}}^{p}\right) \quad p, q \in P, p<q \\
& \left(V_{q}+T\right)-V_{p} \leq T_{\operatorname{tr}}(p, q) \Leftrightarrow \\
& \sum_{p^{\prime}=q}^{p-1} \vec{B}_{p^{\prime}} \geq T-T_{\operatorname{tr}}(p, q)-\sum_{p^{\prime}=q}^{p-1}\left(t_{p^{\prime}}^{s}+t_{p^{\prime}}^{p}\right) \quad p, q \in P, p>q .
\end{aligned}
$$

Adding (19) and (20) when solving (14)-(17) in Step 4 and $4^{\prime}$, and $(14)-(17)+(18)$ in Step $4^{\prime}$ of Algorithm 2 ensures that the algorithm finds the optimal timetable that satisfies the transit time requirements.

This paper focuses on a single round trip/service; hence, our transit time requirements are limited to container flows that use only a single service (unlike the MIP-based approach introduced in Reinhardt et al. 2016). Moreover, like Reinhardt et al. (2016), the requirements are set on the level of the timetable. See Section 7 for a discussion and future research agenda.

\section{Computational Results}

In this section, we will perform a case study with a full factorial sensitivity analysis to test our solution approach. Section 6.1 describes the data used for both the case study and the sensitivity analysis. Next, Section 6.2 describes the case study, and Section 6.3 describes the sensitivity analysis.

\subsection{Data}

To test our method, we use the ME1 route in September 2012 of the Maersk Line network. Time is discretized in units of four hours. Table 2 gives relevant data on this route, which is sailed in a cyclic way from top to bottom. The second column denotes the total (loading and unloading) time planned in each port. The third column gives the actual sailing distance to the next port in the route (obtained from SeaRates 2015). The fourth column shows the scheduled sailing time according to the schedule published by Maersk Line. For example, the scheduled sailing time for Antwerp is 32 hours. Thus, if the actual sailing time between Antwerp and Bremerhaven is 36 hours, then the incurred delay on this sea leg is 4 hours. The fifth column shows the sailing time in hours when the ship sails at maximum speed ( 23 knots). The last column shows the buffer time in the current schedule assuming that the route is sailed at maximum speed. The time needed to make one full round tour is 1,176 hours (seven weeks). Note that this equals the total port time (284 hours) plus the total scheduled sailing time (892 hours). We assume that the route is sailed using a Post-Panamax ship with a capacity of 8,400 20-foot equivalent units (TEUs), using data from
Brouer et al. (2014). The minimum and maximum speed of this ship are 12 and 23 knots $(1 \mathrm{knot}=1 \mathrm{nmi} / \mathrm{hr})$, respectively. Bunker consumption per time unit can be accurately approximated as a constant times the third power of speed. Thus, the fuel cost function becomes

$$
\mathscr{F}_{p}(\tau)=\tilde{b} e\left(\frac{v}{\tilde{v}}\right)^{3} \frac{\left(t_{p}^{s}+\tau\right) l}{24}=\tilde{b} e \frac{\left(t_{p}^{s}+\tau\right) l}{24}\left(\frac{\delta_{p}}{\left(t^{s}+\tau\right) l \tilde{v}}\right)^{3},
$$

where $v$ is the sailing speed in knots, $\delta_{p}$ is the distance in nmi from port $p$ to the next port, and $l=4$ denotes the number of hours in one time unit. The ship has a design speed of $\tilde{v}=16.5$ knots, and bunker consumption at design speed is $\tilde{b}=82.2$ ton per day.

All experiments are run for the model extended with transit time requirements. We allow transit times for each combination of ports on the route to deviate at most 24 hours from the transit time following from the initial schedule, because lengthening the transit by 48 hours is typically acceptable to customers (Reinhardt et al. 2016).

\subsection{Case Study}

6.2.1. Test Instances. Since we do not know the actual delay distribution, we perform experiments for a range of delay distributions. We use uniformly distributed delays for our main case to align with literature (e.g., Wang and Meng 2012a; Li, Qi, and Song 2016): we let each $X_{n}^{s} \sim U\left(0, a+\left\lfloor\delta_{p[n]} / b\right\rfloor\right)$, where $a$ and $b$ are instancespecific parameters and $\delta_{p[n]}$ is the distance between the current and the next port. For the ship to eventually recover from delays, it is important that the average delay per round tour does not exceed the total available buffer of 28 time units. We accordingly construct 10 instances using the parameters $a=\{3,3,2,2,2,1,1,1,0,0\}$ and $b=\{1,200,1,600,800,1,300,2,000,900,1,328,2,400$, $1,000,1,400\}$. These parameters yield an average delay per round tour between 3 and 25.5 time units; see Table 3 . In the table and elsewhere, the expected net buffer time denotes the difference between the total available buffer time and the expected delay per round tour.

For the bunker cost, we use $e=320 \mathrm{USD} /$ ton, which is an estimate of the average bunker cost in 20 global ports from March to August 2017 (ShipAndBunker 2017). The extreme expedite cost for the cut-and-go action is set to 10 million USD per time unit. The delay costs for all ports are $\mathscr{D}_{p}(d)=10,000 d$ for $0 \leq d \leq d_{p}^{\max }=$ 42 time units (one week). We assume that the unit costs exceed 10 million USD for $d>d_{p}^{\text {max }}$, such that delays are bounded by 42 time units. We set the parameters for our algorithm as $w_{p}^{\max }=0.25$ for $p \in P$, $w^{\max }=2$, and $I=25$. Algorithm 2 is implemented exactly as described, except that, to avoid issues because of limited machine precision, we terminate when the difference between $U B$ and $L B$ is smaller than $\epsilon=10^{-8}$ USD. All linear programming models are solved using CPLEX 12.7. 
Table 2. Characteristics of the Route

\begin{tabular}{|c|c|c|c|c|c|}
\hline Port & $\begin{array}{c}\text { Port } \\
\text { time } \\
\text { (hours) }\end{array}$ & $\begin{array}{l}\text { Distance } \\
\text { (nmi) }\end{array}$ & $\begin{array}{l}\text { Scheduled } \\
\text { sailing } \\
\text { time } \\
\text { (hours) }\end{array}$ & $\begin{array}{c}\text { Sailing } \\
\text { time at } 23 \\
\text { knots } \\
\text { (hours) }\end{array}$ & $\begin{array}{c}\text { Buffer } \\
\text { time } \\
\text { (hours) }\end{array}$ \\
\hline Jebel Ali & 31 & 1,329 & 72 & 60 & 12 \\
\hline $\begin{array}{c}\text { Jawaharlal } \\
\text { Nehru }\end{array}$ & 33 & 443 & 24 & 20 & 4 \\
\hline Mundra & 16 & 1,122 & 56 & 52 & 4 \\
\hline Salalah & 14 & 1,553 & 68 & 68 & 0 \\
\hline Jeddah & 11 & 778 & 36 & 36 & 0 \\
\hline Suez Canal & 16 & 2,283 & 100 & 100 & 0 \\
\hline Algeciras & 18 & 1,476 & 88 & 68 & 20 \\
\hline Felixstowe & 24 & 156 & 16 & 8 & 8 \\
\hline Antwerp & 16 & 366 & 32 & 16 & 16 \\
\hline Bremerhaven & 24 & 283 & 24 & 16 & 8 \\
\hline Rotterdam & 20 & 3,829 & 192 & 168 & 24 \\
\hline Suez Canal & 22 & 395 & 20 & 20 & 0 \\
\hline Aqaba & 20 & 656 & 40 & 32 & 8 \\
\hline Jeddah & 19 & 2,648 & 124 & 116 & 8 \\
\hline Totals & 284 & 17,317 & 892 & 780 & 112 \\
\hline
\end{tabular}

6.2.2. Results. For each test instance, we first calculate the cost of the schedule when we consider deterministic delays. That is, we assume that the delay incurred between each two ports is fixed and equal to the expected delay between those two ports. The optimal deterministic schedule is then found by allocating the available buffer time in such a way that a constant speed is used over the round tour. Furthermore, we calculate the costs of the initial schedule as shown in Table 2 and the costs of the optimal schedule using our algorithms.

Table 4 shows the average expected round tour costs, solution times, and number of generated subgradients for the 10 instances. Clearly, the costs of sailing a round tour decrease when the available buffer time increases. The deterministic schedule provides a lower bound on the optimal cost schedule. The difference between the costs of the deterministic and stochastic schedules is the effect of uncertainty on the costs, which is shown in the next two columns of the table. In these columns, first the absolute cost of uncertainty is given and in brackets the relative difference compared with the initial schedule. We observe that for high expected buffer times, a large part of the cost is already incurred in the deterministic case. From the relative costs, we can conclude that the optimal schedule has costs that are $22 \%-71 \%$ lower than the initial schedule. The lowest and largest absolute cost reductions are, respectively, 81 and 192 thousand USD per round tour for 20 and 2.5 time units net expected buffer time. Since liner companies usually provide weekly services, this would result in cost reductions of 4.2-10 million USD per year for this route. The last two columns of Table 4 show the solution time and number of generated subgradients for the instances. All instances can be solved to optimality within 75 seconds, and at most 44 subgradients are computed.

Table 5 shows for each port the probability of arriving on time, the average arrival delay in time units, and the optimal buffer allocation in time units for the instance with an expected net buffer of 20 time units. The table shows that for this case it is impossible to identify a port where the arrival delay is always zero. Note also that more buffer time is added to sea legs with larger distances, because on these legs, larger additional delays are expected to be incurred.

For the same instance, Table 6 shows the sailing times in time units that will be used on the next sea leg given a certain amount of delay. The last columns show the range of feasible speeds and the scheduled sailing time (including buffer time) for the given sea leg. The scheduled sailing time is defined as the sum of the minimum sailing time and the allocated buffer time-i.e., the scheduled sailing time is the time that should be published in the timetable. The table shows that the optimal sailing times are not linear in the delay (see a departure from Antwerp with 0 and 1 time units of delay and a departure from Rotterdam with 4 and 5 time units of delay). Thus, the optimal sailing speed policy is not necessarily to try to recover from all delays during the coming sea leg, in line with Li, Qi, and Song (2016) and contrary to assumptions made in the timetabling literature (e.g., Qi and Song 2012, Wang and Meng 2012b). Furthermore, the table illustrates Lemmas 3 and 4: ships never slow down when they incur higher delays, but higher delays never result in an earlier planned arrival time. Note also that a ship might speed up even when it sails according to schedule: e.g., between Rotterdam and the Suez Canal the scheduled sailing time is 48 time units, but when leaving Rotterdam without delay the optimal speed policy hedges against expected delays on the sea leg by selecting a sailing speed that uses only 47 time units.

\subsection{Sensitivity Analysis}

We next analyze the sensitivity of the results in the previous section by varying several key parameters: the

Table 3. Characteristics of the 10 Instances

\begin{tabular}{lcc}
\hline $\begin{array}{l}\text { Total available buffer } \\
\text { time (time units) }\end{array}$ & $\begin{array}{c}\text { Expected delay per } \\
\text { round tour (time units) }\end{array}$ & $\begin{array}{c}\text { Expected net buffer } \\
\text { time (time units) }\end{array}$ \\
\hline 28.0 & 25.5 & 2.5 \\
28.0 & 23.0 & 5.0 \\
28.0 & 20.5 & 7.5 \\
28.0 & 18.0 & 10.0 \\
28.0 & 15.5 & 12.5 \\
28.0 & 13.0 & 15.0 \\
28.0 & 10.5 & 17.5 \\
28.0 & 8.0 & 20.0 \\
28.0 & 5.5 & 22.5 \\
28.0 & 3.0 & 25.0 \\
\hline
\end{tabular}


Table 4. Total Average Round-Tour Costs for the 10 Test Instances

\begin{tabular}{|c|c|c|c|c|c|c|}
\hline \multirow[b]{2}{*}{$\begin{array}{l}\text { Expected net buffer } \\
\text { (time units) }\end{array}$} & \multirow[b]{2}{*}{$\begin{array}{l}\text { Deterministic schedule } \\
\text { (million USD) }\end{array}$} & \multicolumn{2}{|c|}{ Cost of uncertainty } & \multirow[b]{2}{*}{$\begin{array}{l}\text { Average speed in the deterministic } \\
\text { schedule (knots) }\end{array}$} & \multirow[b]{2}{*}{$\begin{array}{l}\text { Time } \\
\text { (seconds) }\end{array}$} & \multirow[b]{2}{*}{$\begin{array}{l}\text { Number of } \\
\text { subgradients }\end{array}$} \\
\hline & & $\begin{array}{l}\text { Initial buffer } \\
\text { (million USD) }\end{array}$ & $\begin{array}{c}\text { Optimal buffer } \\
\text { (million USD) }\end{array}$ & & & \\
\hline 2.5 & 2.043 & $0.866(100 \%)$ & $0.673(78 \%)$ & 21.9 & 70.0 & 44 \\
\hline 5 & 1.992 & $0.480(100 \%)$ & $0.322(67 \%)$ & 21.6 & 70.2 & 44 \\
\hline 7.5 & 1.944 & $0.373(100 \%)$ & $0.238(64 \%)$ & 21.4 & 38.7 & 19 \\
\hline 10 & 1.897 & $0.298(100 \%)$ & $0.189(63 \%)$ & 21.1 & 43.3 & 20 \\
\hline 12.5 & 1.851 & $0.247(100 \%)$ & $0.152(61 \%)$ & 20.9 & 36.6 & 17 \\
\hline 15 & 1.806 & $0.231(100 \%)$ & $0.140(60 \%)$ & 20.6 & 38.5 & 28 \\
\hline 17.5 & 1.763 & $0.199(100 \%)$ & $0.113(57 \%)$ & 20.4 & 35.5 & 25 \\
\hline 20 & 1.722 & $0.172(100 \%)$ & $0.091(53 \%)$ & 20.1 & 27.8 & 20 \\
\hline 22.5 & 1.682 & $0.176(100 \%)$ & $0.068(38 \%)$ & 19.9 & 42.6 & 30 \\
\hline 25 & 1.643 & $0.147(100 \%)$ & $0.042(29 \%)$ & 19.7 & 22.0 & 19 \\
\hline
\end{tabular}

delay distribution, the mean and standard deviation of the delays, the bunker cost, and the delay cost. Other parameters, as well as algorithm settings, are kept as in the case study. A detailed discussion of the parameters to be varied is given next.

We test the gamma distribution in addition to the uniform delay distribution commonly used in the literature (e.g., Wang and Meng 2012a; Li, Qi, and Song 2016): it models the (unlikely) occurrence of higher delays better and therefore it might be better suitable to represent real disruptions (Mallidis et al. 2018). The gamma distribution is discretized by rounding all fractional realizations to the closest integer value. Each instance uses either gamma or uniform distributions for all legs. For setting the means and standard deviation of the delays, the ports are divided into two groups based on their distance to the next port in the route, where a distance of $1,000 \mathrm{nmi}$ is used as a separator. Parameters for the delay distribution of each group are then chosen in such a way that the means and variances are as shown in the table. For the uniform distribution this means that the lower bound is 0 time units in all three cases and the upper bounds are, respectively, 1, 2, and 3 time units. For the gamma distribution this is done using $\alpha=\{13.5699,1.9359,1.9874\}$, and $\beta=\{0.0378,0.5252,0.7575\}$. In this way, each instance will have one out of nine possible options for the means and standard deviation of port delays.

For the bunker price, we use the estimate of the halfyear average of 20 global ports from the previous sections (320 USD/ton) as the lower bound and the bunker price as used in Brouer et al. (2014) (600 USD/ ton) as the upper bound. Finally, we generated three instances for the delay cost by multiplying the value from the base case by $0.5,1$, and 2, respectively. Table 7 summarizes the varied parameters and associated parameter settings. We construct 162 instances by taking all combinations of settings for all five parameters.

Table 8 presents the results of the sensitivity analysis. Each row of the table represents the average over all instances that share certain parameter values. The fixed parameter values for each row are presented in the first column of the table. Mean 1 and 2 denote, respectively, the mean of the delay distribution for groups 1 (short legs) and 2 (long legs). The next column shows the average deterministic cost in million USD, calculated as in the previous section. Note that in the deterministic schedule, ships are always sailing on time, so only bunker costs are incurred. Furthermore, note that the time $\mathcal{T}$ available for a complete round trip is fixed by the number of ships employed on the route and hence exogenous to our model, which explains why the average speed of the deterministic schedule does not depend on bunker price. The following two columns show the cost of uncertainty for the initial schedule as given in Table 2 and the optimal schedule, respectively. Similarly to the results in the previous section, first the absolute difference is given and in brackets the relative difference with respect to the initial schedule. The next

Table 5. Optimal Buffer Time on the Next Sea Leg for an Expected Net Buffer of 20 Time Units

\begin{tabular}{lcccc}
\hline Port & On time & $\begin{array}{c}\text { Average arrival } \\
\text { delay (time } \\
\text { probability }\end{array}$ & $\begin{array}{c}\text { Distance } \\
\text { (nmi) }\end{array}$ & $\begin{array}{c}\text { Buffer time } \\
\text { (time units) }\end{array}$ \\
\hline Jebel Ali & 0.67 & 0.33 & 1,329 & 1 \\
Jawaharlal & 0.50 & 0.50 & 443 & 1 \\
$\quad$ Nehru & & & & \\
Mundra & 0.50 & 0.50 & 1,122 & 1 \\
Salalah & 0.50 & 0.50 & 1,553 & 2 \\
Jeddah & 0.50 & 0.50 & 778 & 1 \\
Suez Canal & 0.50 & 0.50 & 2,283 & 3 \\
Algeciras & 0.50 & 0.50 & 1,476 & 3 \\
Felixstowe & 0.50 & 0.50 & 156 & 1 \\
Antwerp & 0.75 & 0.25 & 366 & 2 \\
Bremerhaven & 0.88 & 0.13 & 283 & 1 \\
Rotterdam & 0.94 & 0.06 & 3,829 & 6 \\
Suez Canal & 0.67 & 0.33 & 395 & 1 \\
Aqaba & 0.83 & 0.17 & 656 & 1 \\
Jeddah & 0.50 & 0.50 & 2,648 & 4 \\
\hline
\end{tabular}


Table 6. Sailing Time Action in Time Units to Be Used on the Next Sea Leg for an Expected Net Buffer of 20 Time Units

\begin{tabular}{lrrrrrrrrr}
\hline & \multicolumn{8}{c}{ Delay in time units } & Feasible \\
Port & 0 & 1 & 2 & 3 & 4 & 5 & $\geq 6$ & $\begin{array}{c}\text { Scheduled } \\
\text { range }\end{array}$ & sailing time \\
\hline Jebel Ali & 16 & 15 & 15 & 15 & 15 & 15 & 15 & {$[15,27]$} & 16 \\
Jawaharlal & 6 & 5 & 5 & 5 & 5 & 5 & 5 & {$[5,9]$} & 6 \\
$\quad$ Nehru & & & & & & & & & \\
Mundra & 14 & 13 & 13 & 13 & 13 & 13 & 13 & {$[13,23]$} & 14 \\
Salalah & 19 & 18 & 17 & 17 & 17 & 17 & 17 & {$[17,32]$} & 19 \\
Jeddah & 10 & 9 & 9 & 9 & 9 & 9 & 9 & {$[9,16]$} & 10 \\
Suez Canal & 28 & 27 & 26 & 25 & 25 & 25 & 25 & {$[25,47]$} & 28 \\
Algeciras & 20 & 19 & 18 & 17 & 17 & 17 & 17 & {$[17,30]$} & 20 \\
Felixstowe & 2 & 2 & 2 & 2 & 2 & 2 & 2 & {$[2,3]$} & 3 \\
Antwerp & 5 & 5 & 4 & 4 & 4 & 4 & 4 & {$[4,7]$} & 6 \\
Bremerhaven & 4 & 4 & 4 & 4 & 4 & 4 & 4 & {$[4,5]$} & 5 \\
Rotterdam & 47 & 46 & 45 & 44 & 43 & 43 & 42 & {$[42,79]$} & 48 \\
Suez Canal & 5 & 5 & 5 & 5 & 5 & 5 & 5 & {$[5,8]$} & 6 \\
Aqaba & 9 & 8 & 8 & 8 & 8 & 8 & 8 & {$[8,13]$} & 9 \\
Jeddah & 32 & 31 & 30 & 29 & 29 & 29 & 29 & {$[29,55]$} & 33 \\
\hline
\end{tabular}

Notes. For example, when the current delay with respect to the schedule is two time units in Antwerp, the speed on the next sea leg toward Bremerhaven should be set in such a way that the ship will sail this leg in four time units (20 hours).

two columns show the (average) fuel consumption in ton during one round tour. First, the fuel consumption under the deterministic schedule is given and next the average amount of additionally used fuel under uncertainty. The last two columns denote the average solution time in seconds and the average number of subgradients needed to find the optimal solution. The average number of gradients needed is very similar for the instances and varies between 18.8 and 25.9.

We first observe that both the cost of the deterministic schedule and the cost of uncertainty increase when the average delay or the bunker costs increase. When the delay costs increase, the cost of uncertainty also increases. However, the cost of the deterministic schedule is unaffected as delays are never incurred in this schedule. Furthermore, the cost difference between the uniform and gamma delay distributions are quite small. In general, the cost of the deterministic schedule is the same for both distributions. The cost of uncertainty is slightly lower for gamma distributed delays than for uniform distributed delays when having the same mean and variance. When the delay cost increases, one would expect that ships will sail faster to prevent delays and hence burn more fuel. Indeed, we observe that the average additional fuel consumption under uncertainty increases when the delay cost increases. Furthermore, one would expect a reduction in the additional fuel consumption under uncertainty when the bunker cost increases as speeding up will become more expensive in this case. This effect is also visible in the results. The optimal buffer allocation results in schedules that are 3-17 million USD per year cheaper than the initial schedule. The solution times are on average about twice as long when a gamma distribution is used for the delays, and this is because computing $\mathscr{C}^{*}(\vec{B})$ for a single buffer is more time-consuming for the gamma distribution. Average computation time is 70 seconds, and all instances are solved within four minutes.

\section{Conclusion and Future Research}

We developed an approach for buffer allocation in liner shipping that uses a stochastic dynamic program (SDP) for modeling timetable execution-i.e., occurrence and propagation of delays and optimal dynamic recovery actions. Our approach yields the first exact algorithm for optimizing a timetable under stochastic delays, and our algorithm is much faster than algorithms developed using the stochastic programming methodology (e.g., Wang and Meng 2012b). In our experiments, the algorithm computes the optimal buffer time allocation in about 70 seconds. Moreover, in one particular case study we find that the costs of the optimized schedule are 4-10 million USD per year lower than the costs of the initial schedule as executed by Maersk Line. A full factorial experiment with 162 cases shows that this cost improvement is insensitive to model parameters. The experiment also confirms that optimal solutions can be found quickly. Our algorithm is thus exact and very time efficient. Further research investigating possible extensions and limitations of the algorithm is thus of interest.

We next explore such future research directions in the liner shipping domain. Firstly, while our model fixes the number of vessels operating the schedule and thus the total available buffer, optimizing the number of vessels would be straightforward by handling this decision in an outer loop in the algorithm. Another direction of interest is refining our approach for handling transit time requirements. In Section 5.4, we handle these requirements constraints on the published timetable, but alternatively one could include penalties if the actual transit time during timetable execution exceeds the limit. While this extension would be easy to include in our model, we are not sure whether the developed theory extends likewise.

The focus in our paper on a single service is in line with previous literature on stochastic timetabling and recovery actions (Qi and Song 2012; Wang and Meng 2012b; Li, Qi, and Song 2016), and is chiefly motivated

Table 7. Parameter Settings Sensitivity Analysis

\begin{tabular}{ll}
\hline Parameter & \multicolumn{1}{c}{ Values } \\
\hline Delay distribution & Uniform, gamma \\
Delay short legs $\left(\mu ; \sigma^{2}\right)$ (group 1) & $(0.5 ; 0.25),(1.0 ; 0.67),(1.5 ; 1.25)$ \\
Delay long legs $\left(\mu ; \sigma^{2}\right)$ (group 2) & $(0.5 ; 0.25),(1.0 ; 0.67),(1.5 ; 1.25)$ \\
Bunker cost & $320,460,600$ \\
Delay cost & $5,000,10,000,20,000$ \\
\hline
\end{tabular}


Table 8. Average Results per Parameter of the Sensitivity Analysis

\begin{tabular}{|c|c|c|c|c|c|c|c|c|}
\hline \multicolumn{2}{|c|}{$\begin{array}{l}\text { Parameter } \\
\text { setting }\end{array}$} & \multirow[t]{2}{*}{$\begin{array}{l}\text { Deterministic } \\
\text { schedule } \\
\text { (million USD) }\end{array}$} & \multirow[t]{2}{*}{$\begin{array}{l}\text { Cost of uncertainty } \\
\text { for the initial buffer } \\
\text { (million USD) }\end{array}$} & \multirow[t]{2}{*}{$\begin{array}{l}\text { Cost of uncertainty } \\
\text { for the optimal } \\
\text { buffer (million USD) }\end{array}$} & \multirow[t]{2}{*}{$\begin{array}{l}\text { Fuel consumption } \\
\text { under the } \\
\text { deterministic } \\
\text { schedule (ton/ } \\
\text { round tour) }\end{array}$} & \multirow[t]{2}{*}{$\begin{array}{l}\text { Additional fuel } \\
\text { consumption under } \\
\text { uncertainty (ton/ } \\
\text { round tour) }\end{array}$} & \multirow[t]{2}{*}{$\begin{array}{l}\text { Time } \\
\text { (s) }\end{array}$} & \multirow[t]{2}{*}{$\begin{array}{l}\text { Number of } \\
\text { subgradients }\end{array}$} \\
\hline$\mu_{1}$ & $\mu_{2}$ & & & & & & & \\
\hline 0.5 & 0.5 & 2.452 & $0.199(100 \%)$ & $0.095(47 \%)$ & 5,330 & 88 & 39.5 & 19.8 \\
\hline 0.5 & 1.0 & 2.534 & $0.249(100 \%)$ & $0.131(52 \%)$ & 5,509 & 136 & 56.2 & 21.6 \\
\hline 0.5 & 1.5 & 2.622 & $0.317(100 \%)$ & $0.175(55 \%)$ & 5,699 & 167 & 63.9 & 20.1 \\
\hline 1.0 & 0.5 & 2.534 & $0.219(100 \%)$ & $0.119(54 \%)$ & 5,509 & 106 & 48.0 & 19.6 \\
\hline 1.0 & 1.0 & 2.622 & $0.275(100 \%)$ & 0.157 (57\%) & 5,699 & 146 & 75.3 & 18.8 \\
\hline 1.0 & 1.5 & 2.714 & $0.360(100 \%)$ & $0.210(60 \%)$ & 5,899 & 182 & 82.2 & 21.2 \\
\hline 1.5 & 0.5 & 2.622 & $0.258(100 \%)$ & $0.154(59 \%)$ & 5,699 & 128 & 78.7 & 25.9 \\
\hline 1.5 & 1.0 & 2.714 & $0.333(100 \%)$ & $0.201(62 \%)$ & 5,899 & 171 & 90.7 & 22.7 \\
\hline 1.5 & 1.5 & 2.808 & $0.466(100 \%)$ & $0.288(63 \%)$ & 6,104 & 203 & 100.1 & 22.6 \\
\hline \multicolumn{9}{|c|}{ Bunker cost } \\
\hline 32 & & 1.826 & $0.266(100 \%)$ & $0.149(56 \%)$ & 5,705 & 195 & 69.0 & 22.0 \\
\hline 46 & & 2.624 & $0.299(100 \%)$ & $0.172(57 \%)$ & 5,705 & 140 & 68.3 & 20.9 \\
\hline 60 & & 3.423 & $0.327(100 \%)$ & $0.189(57 \%)$ & 5,705 & 108 & 74.2 & 21.1 \\
\hline \multicolumn{9}{|c|}{ Delay cost } \\
\hline & 000 & 2.624 & $0.176(100 \%)$ & $0.100(56 \%)$ & 5,705 & 65 & 76.9 & 20.3 \\
\hline & 1,000 & 2.624 & $0.278(100 \%)$ & $0.162(57 \%)$ & 5,705 & 127 & 71.0 & 21.0 \\
\hline & 1,000 & 2.624 & $0.439(100 \%)$ & $0.248(56 \%)$ & 5,705 & 250 & 63.5 & 22.7 \\
\hline \multicolumn{9}{|c|}{ Distribution } \\
\hline & niform & 2.624 & $0.300(100 \%)$ & $0.174(57 \%)$ & 5,705 & 159 & 42.0 & 20.1 \\
\hline & amma & 2.624 & $0.295(100 \%)$ & $0.166(56 \%)$ & 5,710 & 131 & 99.0 & 22.6 \\
\hline Ove & rall & 2.624 & $0.297(100 \%)$ & $0.170(56 \%)$ & 5,708 & 145 & 70.5 & 21.4 \\
\hline
\end{tabular}

by a desire not to change too many berthing times simultaneously (see Section 3). Recently and in a deterministic setting, Reinhardt et al. (2016) studied timetable optimization for multiple liners simultaneously. As an alternative to optimizing only a single time at once, they introduce a more advanced approach for avoiding the change of too many berthing times: their MIP model includes penalties for each berthing time change. A very interesting direction for future research would be integrating our approach for robust timetabling into their MIP. In particular, the structure of the MIP formulation in Reinhardt et al. (2016) should in principle allow for the insertion of our subgradient-based approach to assess the fuel and delay costs of the individual services. The combined model would yield timetables that are robust against stochastic delays and that satisfy transit time requirements for container flows that use multiple services.

Other interesting avenues for future research are outside of the liner shipping domain. The approach we develop can be extended relatively straightforwardly to optimize the allocation of buffers to the different legs of the published timetable of a single metro, bus, or train line. A key difference here is that for buses, metros, and trains, a long buffer time typically appears after each (round) tour. To see how our approach applies in this case, note that $\mathscr{C}_{1, R|P|+1}^{d e p}\left(d_{1}^{d e p} ; \vec{B}\right)$ represents the expected costs over the first $R$ round tours. Thus, setting $d_{1}^{d e p}=0$ and $R=1$, the problem of allocating buffers to the timetable of a single bus, metro, or tram line can be modeled as $\min _{\vec{B} \in \mathscr{R}} \mathscr{b}_{1,|P|+1}^{d e p}(0 ; \vec{B})$. It is possible (but not trivial) to show that Theorem 2 extends to $\mathscr{C}_{1,|P|+1}^{d e p}(0 ; \vec{B})$, and thus Algorithm 2 can be applied to efficiently solve $\min _{\vec{B} \in \mathscr{B}} \mathscr{C}_{1,|P|+1}^{d e p}(0 ; \vec{B})$. In fact, since $\mathscr{C}_{1,|P|+1}^{d e p}(0 ; \vec{B})$ corresponds to the total costs of a finitehorizon SDP, its evaluation will be at least an order of magnitude faster than evaluating $\mathscr{C}^{*}(\vec{B})$, which corresponds to the long-run average costs. Thus, Algorithm 2 will be even more efficient for optimizing the timetable of single bus, metro, or train lines. It can likewise also be used for optimizing the berthing times of a single execution of a string. However, note that it is not clear how to apply our approach for optimizing the timetable of multiple interacting trains or metros, and this is an interesting direction for future research.

For a final direction of future research, we note that Algorithm 1 indicates a link with submodularity. In particular, similar results may perhaps be obtained via the so-called Lovász extension (Lovász 1983), and this would perhaps lead to a deeper understanding of the results.

\section{Acknowledgments}

The authors thank Twan Dollevoet, Dennis Huisman, Leo Kroon (deceased), Harilaos Psaraftis, Marie Schmidt, Tom van Woensel, and two anonymous referees for valuable comments on the manuscript. 


\section{References}

AhmadBeygi S, Cohn A, Lapp M (2010) Decreasing airline delay propagation by re-allocating scheduled slack. IIE Trans. 42(7): 478-489.

Aktürk M, Atamtürk A, Gürel S (2014) Aircraft rescheduling with cruise speed control. Oper. Res. 62(4):829-845.

Arikan U, Gürel S, Aktürk M (2016) Integrated aircraft and passenger recovery with cruise time controllability. Ann. Oper. Res. 236(3): 295-317.

Bertsekas D (2007) Dynamic Programming and Optimal Control, vol. 2, 3rd ed. (Athena Scientific, Belmont, MA).

Binder A, Albrecht T (2012) Predictive energy-efficient running time control for metro lines. CASPT12 Conf. Advanced Systems Public Transport, Santiago, Chile.

Brouer B, Álvarez J, Plum C, Pisinger D, Sigurd M (2014) A base integer programming model and benchmark suite for linershipping network design. Transportation Sci. 48(2):281-312.

Brouer B, Dirksen J, Pisinger D, Plum C, Vaaben B (2013) The vessel schedule recovery problem (VSRP)—A MIP model for handling disruptions in liner shipping. Eur. J. Oper. Res. 224(2):362-374.

CargoSmart (2017) Schedule reliability: September snapshot. Accessed November 7, 2017, https://www.cargosmarton.com/schedulereliability-september-snapshot\#more-6777.

Cariou P (2011) Is slow steaming a sustainable means of reducing $\mathrm{CO}_{2}$ emissions from container shipping? Transportation Res. Part D: Transport Environ. 16(3):260-264.

Chiraphadhanakul V, Barnhart C (2013) Robust flight schedules through slack re-allocation. EURO J. Transportation Logist. 2(4): 277-306.

Christiansen M, Fagerholt K, Ronen D (2004) Ship routing and scheduling: Status and perspectives. Transportation Sci. 38(1): $1-18$.

Cicerone S, Di Stefano G, Schachtebeck M, Schöbel A (2012) Multistage recovery robustness for optimization problems: A new concept for planning under disturbances. Inform. Sci. 190:107-126.

Cicerone S, D'Angelo G, Di Stefano G, Frigiono D, Navarra A, Schachtebeck M, Schöbel A (2009) Recoverable robustness in shunting and timetabling. Ahuja R, Möhring R, Zaroliagis C, eds. Robust and Online Large-Scale Optimization, Lecture Notes in Computer Science, vol. 5868 (Springer, Berlin), 28-60.

Clausen J, Larsen A, Larsen J, Rezanova N (2010) Disruption management in the airline industry-Concepts, models and methods. Comput. Oper. Res. 37(5):809-821.

Corman F, D'Ariano A, Pacciarelli D, Pranzo M (2010) A tabu search algorithm for rerouting trains during rail operations. Transportation Res. Part B: Methodology 44(1):175-192.

Cucala A, Fernández A, Sicre C, Domínguez M (2012) Fuzzy optimal schedule of high speed train operation to minimize energy consumption with uncertain delays and driver's behavioral response. Engrg. Appl. Artificial Intelligence 25(8):1548-1557.

Du Y, Meng Q, Wang Y (2015) Budgeting the fuel consumption of a container ship over a round voyage via robust optimization. Transportation Res. Rec. J. Transportation Res. Board 2477:68-75.

Ducruet C, Notteboom T (2012) Developing liner service networks in container shipping. Song D, Panayides P, eds. Maritime Logistics: A Complete Guide to Effective Shipping and Port Management (Kogan Page, London), 77-100.

Duran A, Gürel S, Aktürk M (2015) Robust airline scheduling with controllable cruise times and chance constraints. IIE Trans. 47(1):64-83.

Engelhardt-Funke O, Kolonko M (2004) Analysing stability and investments in railway networks using advanced evolutionary algorithms. Internat. Trans. Oper. Res. 11(4):381-394.

Fischetti M, Salvagnin D, Zanette A (2009) Fast approaches to improve the robustness of a railway timetable. Transportation Sci. 43(3):321-335.
Franceschetti A (2015) Algorithms for the departure times and speed optimization problems. Sustainable city logistics: Fleet planning, routing and scheduling problems, Chapter 5. PhD thesis, Eindhoven University of Technology, Eindhoven, Netherlands.

Gong C, Zhang S, Zhang F, Jiang J, Wang X (2014) An integrated energyefficient operation methodology for metro systems based on a real case of Shanghai Metro Line One. Energies 7(11):7305-7329.

Hassannayebi E, Sajedinejad A, Mardani S (2014) Urban rail transit planning using a two-stage simulation-based optimization approach. Simul. Model. Practice Theory 49:151-166.

Hvattum L, Norstad I, Fagerholt K, Laporte G (2013) Analysis of an exact algorithm for the vessel speed optimization problem. Networks 62(2):132-135.

Khachiyan LG (1980) Polynomial algorithms in linear programming. USSR Comput. Math. Math. Phys. 20(1):53-72.

Kramer R, Maculan N, Subramanian A, Vidal T (2015) A speed and departure time optimization algorithm for the pollution-routing problem. Eur. J. Oper. Res. 247(3):782-787.

Kroon L, Dekker R, Vromans M (2007) Cyclic railway timetabling: A stochastic optimization approach. Geraets F, Kroon L, Schöbel A, Wagner D, Zaroliagis C, eds. Algorithmic Methods for Railway Optimization, Lecture Notes in Computer Science, vol. 4359 (Springer, Berlin), 41-66.

Kroon L, Maróti G, Retel Helmrich M, Vromans M, Dekker R (2008) Stochastic improvement of cyclic railway timetables. Transportation Res. Part B: Methodology 42(6):553-570.

Lan S, Clarke J, Barnhart C (2006) Planning for robust airline operations: Optimizing aircraft routings and flight departure times to minimize passenger disruptions. Transportation Sci. 40(1):15-28.

Li C, Qi X, Lee C (2015) Disruption recovery for a vessel in liner shipping. Transportation Sci. 49(4):900-921.

Li C, Qi X, Song D (2016) Real-time schedule recovery in liner shipping service with regular uncertainties and disruption events. Transportation Res. Part B: Methodology 93(Part B):762-788.

Liebchen C, Schachtebeck M, Schöbel A, Stiller S, Prigge A (2010) Computing delay resistant railway timetables. Comput. Oper. Res. 37(5):857-868.

Lovász L (1983) Submodular functions and convexity. Bachem A, Korte B, Grötschel M, eds. Mathematical Programming-The State of the Art, Bonn 1982 (Springer, Berlin), 235-257.

Maher S (2015) Solving the integrated airline recovery problem using column-and-row generation. Transportation Sci. 50(1):216-239.

Mallidis I, Iakovou E, Dekker R, Vlachos D (2018) The impact of slow steaming on the carriers' and shippers' costs: The case of a global logistics network. Transportation Res. Part E: Logist. Transportation Rev. 111:18-39.

Mulder J, Dekker R (2018) Optimisation in container liner shipping. Geerlings H, Kuipers B, Zuidwijk R, eds. Ports and Networks: Strategies, Operations and Perspectives (Taylor \& Francis Group, Routledge, London), 181-203.

Nesterov Y (2013) Introductory Lectures on Convex Optimization: A Basic Course, vol. 87 (Springer Science \& Business Media, New York).

Norlund E, Gribkovskaia I, Laporte G (2015) Supply vessel planning under cost, environment and robustness considerations. Omega 57(Part B):271-281.

Notteboom T, Vernimmen B (2009) The effect of high fuel costs on liner service configuration in container shipping. J. Transport Geography 17(5):325-337.

Petersen J, Sölveling G, Clarke J, Johnson E, Shebalov S (2012) An optimization approach to airline integrated recovery. Transportation Sci. 46(4):482-500.

Psaraftis H, Kontovas C (2013) Speed models for energy-efficient maritime transportation: A taxonomy and survey. Transportation Res. Part C: Emerging Tech. 26:331-351.

Qi X, Song D (2012) Minimizing fuel emissions by optimizing vessel schedules in liner shipping with uncertain port times. 
Transportation Res. Part E: Logist. Transportation Rev. 48(4): 863-880.

Reinhardt LB, Plum C, Pisinger D, Sigurd M, Vial G (2016) The liner shipping berth scheduling problem with transit times. Transportation Res. Part E: Logist. Transportation Rev. 86:116-128.

Rosenberger J, Johnson E, Nemhauser G (2003) Rerouting aircraft for airline recovery. Transportation Sci. 37(4):408-421.

SeaRates (2015) Reference guide, port to port distance. Accessed October 19, 2015, https://www.searates.com/reference/portdistance.

Ship \& Bunker (2017) Global 20 ports average bunker costs for the last 6 months. Accessed August 17, 2017, https://shipandbunker. $\mathrm{com} / \mathrm{prices} / \mathrm{av} / \mathrm{global} / \mathrm{av}$-g20-global-20-ports-average.

Visentini M, Borenstein D, Li J, Mirchandani P (2014) Review of realtime vehicle schedule recovery methods in transportation services. J. Scheduling 17(6):541-567.
Wang S, Meng Q (2012a) Liner ship route schedule design with sea contingency time and port time uncertainty. Transportation Res. Part B: Methodology 46(5):615-633.

Wang S, Meng Q (2012b) Robust schedule design for liner shipping services. Transportation Res. Part E: Logist. Transportation Rev. 48(6):1093-1106.

Wu C (2005) Inherent delays and operational reliability of airline schedules. J. Air Transport Management 11(4):273-282.

Wu C, Caves $\mathrm{R}$ (2003) The punctuality performance of aircraft rotations in a network of airports. Transportation Planning Tech. 26(5): 417-436.

Wu Y, Tang J, Yu Y, Pan Z (2015) A stochastic optimization model for transit network timetable design to mitigate the randomness of traveling time by adding slack time. Transportation Res. Part C: Emerging Tech. 52:15-31. 